\title{
Neutron and ARPES Constraints on the Couplings of the Multiorbital Hubbard Model for the Pnictides
}

\author{
Qinlong Luo, ${ }^{1,2}$ George Martins, ${ }^{3}$ Dao-Xin Yao, ${ }^{1,2,4}$ Maria Daghofer, ${ }^{5}$ Rong Yu, ${ }^{6}$ Adriana Moreo,,${ }^{1,2}$ and Elbio Dagotto ${ }^{1,2}$ \\ ${ }^{1}$ Department of Physics and Astronomy, University of Tennessee, Knoxville, Tennessee 37996, USA \\ ${ }^{2}$ Materials Science and Technology Division, Oak Ridge National Laboratory, Oak Ridge, Tennessee 32831, USA \\ ${ }^{3}$ Physics Department, Oakland University, Rochester, Michigan 48309, USA \\ ${ }^{4}$ State Key Laboratory of Optoelectronic Materials and Technologies, \\ School of Physics and Engineering, Sun Yat-Sen University, Guangzhou 510275, China \\ ${ }^{5}$ IFW Dresden, P.O. Box 2701 16, D-01171 Dresden, Germany \\ ${ }^{6}$ Department of Physics and Astronomy, Rice University, Houston, Texas 77005, USA
}

(Dated: November 3, 2018)

\begin{abstract}
The results of neutron scattering and angle-resolved photoemission experiments for the Fe-pnictide parent compounds, and their metallic nature, are shown to impose severe constraints on the range of values that can be considered "realistic" for the intraorbital Hubbard repulsion $U$ and Hund coupling $J$ in multiorbital Hubbard models treated in the mean-field approximation. Phase diagrams for three- and five-orbital models are here provided, and the physically realistic regime of couplings is highlighted, to guide future theoretical work into the proper region of parameters of Hubbard models. In addition, using the random phase approximation, the pairing tendencies in these realistic coupling regions are investigated. It is shown that the dominant spin-singlet pairing channels in these coupling regimes correspond to nodal superconductivity, with strong competition between several states that belong to different irreducible representations. This is compatible with experimental bulk measurements that have reported the existence of nodes in several Fe-pnictide compounds.
\end{abstract}

PACS numbers: 74.70.Xa, 74.20.-z, 74.20.Rp

\section{INTRODUCTION}

An exciting new area of research has recently opened with the discovery of superconductivity in the Fe pnictides $\underline{\underline{1}-\underline{\underline{7}}}$ These materials present many similarities with the high temperature superconductors based on copper, but also several differences. Both families have layered structures suggesting that theoretical studies in two dimensional lattices should be able to capture the essence of the pairing mechanism. In the undoped limit, both types of compounds are magnetic, with wavevector $(\pi, 0)$ in the case of the pnictides ${ }^{8.9}$ and $(\pi, \pi)$ for the cuprates, in the notation of the square lattice defined by $\mathrm{Fe}$ or $\mathrm{Cu}$. However, a crucial difference is that the undoped parent compound is an insulator for the cuprates, while it is a (bad) metal for the pnictides. This fact already suggests that the regime of a large Hubbard coupling $U$, widely used in the context of the cuprates, may not be appropriate for a theoretical description of the pnictides.

Adding to the complexity of this problem, it is also clear that a theoretical study of pnictides cannot rely on just one orbital, as in the case of the cuprates, but it needs a multiorbital approach $\stackrel{10}{=}$ In fact, to study the magnetic and superconducting properties of the pnictides, a considerable effort has already started using multiorbital model Hamiltonians $\stackrel{11-33}{\underline{12}}$ Having to consider multiple orbitals severely restricts the available tools to carry out unbiased computer-based investigations of Hubbard multiorbital models. As a consequence, several studies have been restricted to mean-field approximations. Fortunately, this is not a drastic limitation for the case of the undoped systems, since similar mean-field approximations for the cuprates are known to capture qualitatively the essence of the magnetic states $\frac{34}{4}$ In fact, recent mean-field-based efforts for the pnictides have already reported the presence of a state that is simultaneously metallic and magnetic. $\frac{18}{}$ However, the study of the intermediate coupling regime, where the magnetic-metallic state was found, establishes a considerable challenge to theory since an intermediate region of couplings is often more difficult to analyze than either extreme of large or small $U$. In addition, a multiorbital Hubbard approach needs at least two couplings: the on-site intra-orbital Hubbard repulsion $U$ and the on-site Hund coupling $J$. (A third parameter, the on-site inter-orbital repulsion $U^{\prime}$, is then defined by the well-known relation $U=U^{\prime}+2 J$ arising from symmetries in orbital space $\frac{35}{5}$ ) Having two couplings increases further the complexity of the analysis and the comparison between different approaches since there is at present no universally accepted range of $U$ and $J$ that is considered realistic by the community of experts. In fact, it would be quite desirable to restrict the values of the $U$ and $J$ couplings used in the literature to a much narrower range, where qualitative agreement with experiments is observed.

In this publication, our goal is to use experimental neutron scattering and photoemission data for the undoped pnictide parent compounds, supplemented by their well-known metallic properties, to establish lower and upper bounds on the couplings $U$ and $J$ of multiorbital Hubbard models. By focusing on a more restricted set of couplings, our results will guide future theoretical efforts into a realistic regime for the pnictides. Our calculations are based on the previously used and tested mean-field approximation,,$\frac{18}{}$ allowing us to calculate a variety of observables that are then compared against experimental results to establish the proper ranges for $U$ and $J$.

To carry out this theory-experiment comparison the focus here is on the results of two powerful experimental techniques. One of them is neutron scattering. In these experiments, a magnetic order with wavevector $(\pi, 0)$ (in the Fe square-lattice 
notation) was observed at low temperatures $\frac{8.9}{2}$ This particular wavevector can be theoretically accommodated by merely using proper tight-binding model Hamiltonians that reproduce the well-known paramagnetic Fermi surface (with hole and electron pockets). More important for our purposes is the actual value of the ordered moment for the iron spin, $\mu_{\mathrm{Fe}}$. Table 2 of Ref. 8 and Table 2 of Ref. 9 provide a summary of the neutron scattering experimental values for $\mu_{\mathrm{Fe}}$ : within the 1111 and 122 families they range from 0.25 for NdOFeAs to $\sim 1$ for $\mathrm{SrFe}_{2} \mathrm{As}_{2}$ and $\mathrm{BaFe}_{2} \mathrm{As}_{2}$ (the 11 family has substantially larger values of $\mu_{\mathrm{Fe}}$, but their ordering wavevectors are different, indicating that the 11 compounds require a special discussion beyond the scope of this work). Since the mean-field investigations reported here focus on the mean-field solution corresponding to wavevector $(\pi, 0)$, the theoretical value of $\mu_{\mathrm{Fe}}$ can be obtained when varying the couplings in the Hubbard model, and a range compatible with experiments can be found. Not surprisingly, considering the (bad) metallic character of these compounds, the range of interest will be shown to correspond to intermediate values of the on-site repulsion $U$, as also discussed before ${ }^{18,25}$ However, our investigations below show that sizable constraints over the Hund coupling $J$, not explored before, can also be obtained.

Another powerful experimental technique that will help us to establish constraints on the couplings of the Hubbard models is Angle Resolved Photoemission (ARPES). Applying this technique, several reports describing the Fermi surface of the pnictides have been presented, including their temperature evolution. Here, the vast ARPES literature will not be comprehensively reviewed but the focus will be on the results for the undoped compounds in the spin density wave (SDW) regime. In particular, it has been reported by several groups that in this magnetically ordered state the Fermi surface presents extra "features" in the vicinity of the $\Gamma$-point hole pockets that are not present in band-structure calculations for the paramagnetic state. These extra features, mainly found for the 122 compounds, are described as the existence of "satellite" pockets caused by $V$-shaped bands (or by $V$ shaped band crossings) $36-42$ Our focus will be on trying to reproduce qualitatively with mean-field approximations these type of satellite extra features that ARPES investigations have systematically reported in the magnetic state. Note that near the original electron pockets at $(\pi, 0)$ and $(0, \pi)$ there are also several reports of interesting modifications of the paramagnetic band structure when at low temperatures. However, in our opinion, the ARPES analysis of the modifications to the band structure electron pockets appears less robust due to the presence of considerable noise in the raw data, more than for the case of the hole pockets. For this reason, the focus here will be on the existence of satellite pockets at low temperatures near the original $\Gamma$-point hole-pockets. Note also that these satellite features have been given an electron-like character in some publications ${ }^{38}$ but considering the uncertainties in ARPES studies, mainly caused by the considerable backgrounds, our analysis below will focus on finding regions in the $U-J / U$ plane where any kind of extra pocket is induced by the magnetic order close to the original $\Gamma$-point hole-pockets, regardless of whether its character is electron- or hole-like.
While finding constraints on $U$ and $J$ in multiorbital Hubbard models is already interesting, our effort here continues with the analysis of the pairing states that are obtained in those "realistic" $U-J / U$ regions, via the use of the Random Phase Approximation (RPA) ${ }^{21,22}$ Exploring pairing tendencies is important since there is a growing controversy in the Fe pnictides investigations with regards to the symmetry of the superconducting order parameter. While a variety of photoemission experiments report the absence of nodes, ${ }^{43-48}$ at least clearly with regards to the $\Gamma$-point hole pockets, other bulk experiments report results compatible with a nodal superconducting state. ${ }^{49-60}$ This controversy has not been resolved and it is one of the most important open problems in pnictides. Our investigations show that in the realistic $U-J / U$ regimen identified here, for both three- and five-orbital models, the RPA dominant superconducting state is nodal (this includes the $A_{1 g}$ state with zeros (or near zeros) of the order parameter). Moreover, within RPA there is a clear competition between a variety of states belonging to different irreducible representations suggesting that different pnictides may have different symmetries with regards to the superconducting order parameter. All these competing states are nodal or quasi-nodal, at least within the limitations of our approximations.

The organization of this manuscript is the following. First, the models and technique used are presented in Sec. [II. This is followed in Sec. III by the search for a realistic $U-J / U$ regime for the three-orbital model. The case of five orbitals is presented in Sec. IV. The dominant RPA pairing tendencies in the realistic regime are presented in Sec. V. Finally, conclusions are provided in Sec.VI

\section{MODELS AND TECHNIQUES}

\section{A. The model Hamiltonians}

In this effort, the three-orbital Hubbard model introduced in Ref. 31 will be used first. This model is purely based on the $d$ electrons of Fe and it considers only the three orbitals $d_{x z}$, $d_{y z}$, and $d_{x y}$, widely believed to be the most relevant orbitals at the Fermi surface for the pnictides. The reader is referred to the original publication ${ }^{31}$ for a full description of the model and its band structure (which is in good agreement with abinitio calculations). In momentum space, the model includes a tight-binding term defined as:

$$
H_{\mathrm{TB}}(\mathbf{k})=\sum_{\mathbf{k}, \sigma, \mu, v} T^{\mu, v}(\mathbf{k}) d_{\mathbf{k}, \mu, \sigma}^{\dagger} d_{\mathbf{k}, v, \sigma},
$$

with

$$
\begin{aligned}
T^{11}= & 2 t_{2} \cos k_{x}+2 t_{1} \cos k_{y}+4 t_{3} \cos k_{x} \cos k_{y}-\mu, \\
T^{22}= & 2 t_{1} \cos k_{x}+2 t_{2} \cos k_{y}+4 t_{3} \cos k_{x} \cos k_{y}-\mu, \\
T^{33}= & 2 t_{5}\left(\cos k_{x}+\cos k_{y}\right) \\
& +4 t_{6} \cos k_{x} \cos k_{y}-\mu+\Delta_{x y}, \\
T^{12}= & T^{21}=4 t_{4} \sin k_{x} \sin k_{y}, \\
T^{13}= & \bar{T}^{31}=2 i t_{7} \sin k_{x}+4 i t_{8} \sin k_{x} \cos k_{y},
\end{aligned}
$$


TABLE I: Parameters for the tight-binding portion of the threeorbital model used here. The overall energy unit is electron volts.

\begin{tabular}{|ccccccccc|}
\hline$t_{1}$ & $t_{2}$ & $t_{3}$ & $t_{4}$ & $t_{5}$ & $t_{6}$ & $t_{7}$ & $t_{8}$ & $\Delta_{x y}$ \\
\hline 0.02 & 0.06 & 0.03 & -0.01 & 0.2 & 0.3 & -0.2 & $-t_{7} / 2$ & 0.4 \\
\hline
\end{tabular}

$$
T^{23}=\bar{T}^{32}=2 i t_{7} \sin k_{y}+4 i t_{8} \sin k_{y} \cos k_{x},
$$

where a bar on top of a matrix element denotes the complex conjugate. $\mu$ and $v$ range from 1 to 3 and label the orbitals $d_{x z}$ (1), $d_{y z}(2)$, and $d_{x y}$ (3). Since the Hamiltonian for a one-iron unit cell is here considered, then $\mathbf{k}$ runs within the corresponding extended Brillouin zone (BZ) $-\pi<k_{x}, k_{y} \leq \pi$. The actual values for the hopping amplitudes are in Table I.

The Coulombic interacting portion of the three-orbital Hamiltonian is given by:

$$
\begin{aligned}
H_{\text {int }}= & U \sum_{\mathbf{i}, \alpha} n_{\mathbf{i}, \alpha, \uparrow} n_{\mathbf{i}, \alpha, \downarrow}+\left(U^{\prime}-J / 2\right) \sum_{\mathbf{i}, \alpha<\beta} n_{\mathbf{i}, \alpha} n_{\mathbf{i}, \beta} \\
& -2 J \sum_{\mathbf{i}, \alpha<\beta} \mathbf{S}_{\mathbf{i}, \alpha} \cdot \mathbf{S}_{\mathbf{i}, \beta} \\
& +J \sum_{\mathbf{i}, \alpha<\beta}\left(d_{\mathbf{i}, \alpha, \uparrow}^{\dagger} d_{\mathbf{i}, \alpha, \downarrow}^{\dagger} d_{\mathbf{i}, \beta, \downarrow} d_{\mathbf{i}, \beta, \uparrow}+\text { h.c. }\right),
\end{aligned}
$$

where $\alpha, \beta=1,2,3$ denote the orbitals, $\mathbf{S}_{\mathbf{i}, \alpha}\left(n_{\mathbf{i}, \alpha}\right)$ is the spin (electronic density) of orbital $\alpha$ at site $\mathbf{i}$ (this index labels sites of the square lattice defined by the irons), and the relation $U^{\prime}=U-2 J$ between the Kanamori parameters has been used. $^{61}$ The first two terms give the energy cost of having two electrons located in the same orbital or in different orbitals, both at the same site, respectively. The second line contains the Hund's rule coupling that favors the ferromagnetic (FM) alignment of the spins in different orbitals at the same lattice site. The "pair-hopping" term is in the third line and its coupling is equal to $J$ by symmetry. Note that the values used for $U$ and $J$ can be substantially smaller than the atomic ones, because the interactions may be screened by bands not included in the Hamiltonian. The Coulombic interaction terms introduced above have been used and discussed in several previous publications $13,18,20,25,31$ where more details can be found by the readers. All energies are provided here in electron volts. As shown in Ref. 31, the electronic density of relevance for this model is $n=4$ to reproduce the expected Fermi surface in the paramagnetic regime.

In the present investigation, two five-orbital models (also based only on the $d$ electrons of $\mathrm{Fe}$ ) have also been used, at electronic density $n=6$. By supplementing the three-orbital model by more complicated five orbital versions, our main goal is to verify the self-consistency of our approach. In other words, if the many models, with similar Fermi surfaces by construction, would give quite different ranges of couplings for the compatibility with neutron and ARPES results, then this would raise concerns about the entire calculation. It turns out that, as shown below, the $J / U$ and $U$ ranges that are found to be physically reasonable are similar in all cases, demonstrating that our approach is self-consistent. With regards to the specific five-orbital models used here, the tight-binding parameters of one of them are in the Appendix, while another set of hoppings is from Ref. 21. At $U=0$, all these models provide a Fermi surface (see below) that compares well with experiments and band structure calculations for the $122 \mathrm{com}-$ pounds. The Coulombic interactions for five-orbitals are the obvious generalization of the terms used for three-orbitals.

\section{B. The mean-field approximation}

To study the ground state properties of the models introduced before, a mean-field approximation will be applied. This approximation was already presented in previous publications, $\stackrel{18,25}{ }$ but it is here also discussed for completeness. The simple standard assumption of considering only the mean-field values for the diagonal operators is followed: ${ }^{62}$

$$
\left\langle d_{\mathbf{i}, \mu, \sigma}^{\dagger} d_{\mathbf{j}, v, \sigma^{\prime}}\right\rangle=\left(n_{\mu}+\frac{\sigma}{2} \cos \left(\mathbf{q} \cdot \mathbf{r}_{\mathbf{i}}\right) m_{\mu}\right) \delta_{\mathbf{i j}} \delta_{\mu \nu} \delta_{\sigma \sigma^{\prime}}
$$

where $\mathbf{q}$ is the ordering wavevector of the magnetic order. $n_{\mu}$ and $m_{\mu}$ are mean-field parameters (to be determined selfconsistently) describing the charge density and magnetization of the orbital $\mu$, respectively. The rest of the notation is standard. Applying Eq. (9) to $H_{\text {int }}$, the mean-field Hamiltonian in momentum space can be written as

$$
\begin{array}{r}
H_{\mathrm{MF}}=H_{\mathrm{TB}}+C+\sum_{\mathbf{k}, \mu, \sigma} \epsilon_{\mu} d_{\mathbf{k}, \mu, \sigma}^{\dagger} d_{\mathbf{k}, \mu, \sigma} \\
+\sum_{\mathbf{k}, \mu, \sigma} \eta_{\mu, \sigma}\left(d_{\mathbf{k}, \mu, \sigma}^{\dagger} d_{\mathbf{k}+\mathbf{q}, \mu, \sigma}+d_{\mathbf{k}+\mathbf{q}, \mu, \sigma}^{\dagger} d_{\mathbf{k}, \mu, \sigma}\right),
\end{array}
$$

where $\mathbf{k}$ runs over the extended first $\mathrm{BZ}, H_{\mathrm{TB}}$ is the hopping term in Eq. (1), the constant $C$ is

$$
\begin{aligned}
C= & -N U \sum_{\mu}\left(n_{\mu}^{2}-\frac{1}{4} m_{\mu}^{2}\right)-N\left(2 U^{\prime}-J\right) \sum_{\mu \neq v} n_{\mu} n_{v} \\
& +\frac{N J}{2} \sum_{\mu \neq v} m_{\mu} m_{v},
\end{aligned}
$$

$N$ is the number of sites, and the following definitions were introduced

$$
\begin{gathered}
\epsilon_{\mu}=U n_{\mu}+\left(2 U^{\prime}-J\right) \sum_{v \neq \mu} n_{v}, \\
\eta_{\mu, \sigma}=-\frac{\sigma}{2}\left(U m_{\mu}+J \sum_{v \neq \mu} m_{v}\right) .
\end{gathered}
$$

The mean-field Hamiltonian can be numerically solved for a fixed set of mean-field parameters using standard library subroutines. $n_{\mu}$ and $m_{\mu}$ are obtained self-consistently by minimizing the energy via an iterative process. During the iterations $\sum_{\mu} n_{\mu}=n$ was enforced at each step, such that the total charge density is a constant (4 for the three-orbital model, and 6 for the five-orbital models). The reader should assume that these are the electronic densities used for these models 
throughout the manuscript, both in the mean-field approximation and for the RPA approximation as well. Note also that the numerical solution of the mean-field Hamiltonian immediately allows for the calculation of the band structure, density of states (DOS), and magnetization $\left(m=\sum_{\mu} m_{\mu}\right)$ at the ordering wavevector q. Moreover, the photoemission spectral function can also be calculated, as explained in Ref. 18.

\section{RESULTS FOR THE THREE-ORBITAL MODEL}

Our discussion of results starts with the three-orbital model. Some aspects of this discussion have been briefly mentioned in other publications, thus references to those previous efforts are provided where appropriate.

\section{A. Comparison with neutron scattering experiments}

Figure 1 contains the mean-field order parameter $(m)$ at wavevector $(\pi, 0)$ vs. $U$. The plotted values for $m$ arise from the numerical solution of the mean-field equations discussed in the previous section. For small values of $J / U$, such as 0.00 and 0.05 , in Fig. $1 m$ discontinuously jumps from zero to a robust value at a critical $U$. While such a discontinuity is observed in all models discussed in this paper, its origin is not universal. It is caused by a metal-insulator transition in a fourband model, see Sec. II.C.4 of Ref. 18. In the three-orbital model, the discontinuity only coincides with the opening of a gap for smaller $J / U \lesssim 0.15$, and is rather marked by the sudden onset of strong orbital order, with a close competition between substantial alternating and nearly perfect ferro-orbital order. ${ }^{31}$ For larger $\mathrm{a} 0.15 \lesssim J / U \lesssim 0.22$, states with both types of orbital order can remain metallic for a small range of $U$ just above the onset of strong orbital order, but the FS is qualitatively very different from ARPES results, e.g. it does not feature any sign of hole pockets around the $\Gamma$ point. ${ }^{31}$ At or rapidly after the critical $U$, the density of states develops a gap (not shown), signaling insulating behavior, in contradiction with the experimentally observed (bad) metallic character of the undoped pnictides. Moreover, as a consequence of the discontinuity, the order parameter $m$ in the range of small $J / U$ never reaches the realistic values for pnictides reported in neutron scattering experiments, i.e. $[0.25,1.0]$ for $(\pi, 0)$ magnetic order. Thus, these results for small $J / U$ start illustrating one of the main messages of this publication, namely that within the mean-field approximation used in our effort the request of qualitative agreement with the experimental properties of the undoped pnictides imposes severe constraints on the values of $U$ and $J / U$ for the multi-orbital Hubbard models. In particular, it is clear that $J / U=0.00$ and 0.05 do not seem physically appropriate to describe the pnictides.

As $J / U$ increases further, $m$ now develops (becomes nonzero) at an earlier critical value of $U$, allowing for a proper description of materials with weak magnetic order parameters such as the "1111" family. Note that for $J / U=0.10,0.15$, and 0.20 , a discontinuity is still present in $m$ vs. $U$, so not all values of the order parameter $m$ are possible, while for larger

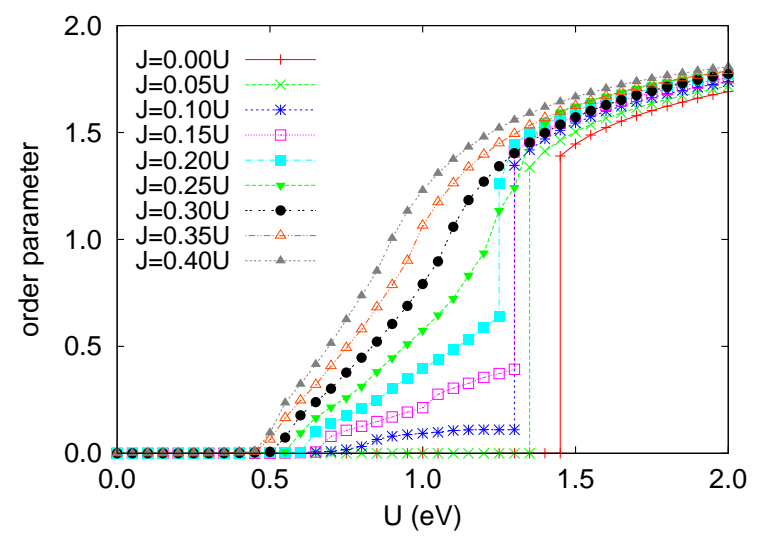

FIG. 1: Mean-field order parameter at wavevector $(\pi, 0)$ vs. $U$ (in eV units) for the three-orbital model discussed in the text, and parametric with the values of $J / U$ indicated.

$J / U$ s the $m$ curves are no longer discontinuous. $J / U=0.50$ is the largest ratio that should be considered to avoid a negative $U^{\prime}$ due to the relation $U=U^{\prime}+2 J$. Thus, adding this information to the results for $m$ and its comparison with neutron scattering, the proper range of $J / U$ couplings naively becomes $\sim[0.10,0.50]$, with $U$ larger than the first critical value where $m$ develops. However, if in addition it is considered that $J$ should be smaller than $U^{\prime}$, then this reduces the range further to $[0.10,0.33]$, since $J=U^{\prime}$ at $J / U=1 / 3$.

\section{B. Comparison with ARPES experiments}

As discussed in the Introduction, another experimental source of information that can be used to reduce the allowed range of couplings in the Hubbard model is provided by the ARPES results for undoped pnictides. As previously mentioned, a common generic feature of several ARPES experiments at low temperatures in the SDW phase is the development of "extra" features (pockets) near the original $\Gamma$-point hole pockets of the noninteracting limit. To search for these features, within our mean-field approximation the one-particle spectral function $A(\mathbf{k}, \omega)$ has been calculated, and the Fermi surface results have been analyzed in a wide range of $U$ and $J / U$, using a $\delta$-function broadening $0.025 \mathrm{eV}$.

Shown in Fig. 2 are representative results of our ARPES calculations (see also Refs. 31,33). The focus is on a range of $J / U$ and $U$ where mean-field ARPES contains a $\Gamma$-point hole pocket, as in the original $U=0$ bandstructure (presumably corresponding to the high temperature non-magnetic regime as well), and in addition "satellite" pockets as in ARPES, in between the original hole and electron pockets along the $k_{x}$ axis. The results have not been folded, but are representative of a single-domain spin order wavevector, in this case $(\pi, 0)$, and with only one Fe per unit cell. Figure 2 shows representative cases where these satellites are clearly present [the satellite pockets tend to be electron-like for small $U$, switching to hole-like for slightly larger, but still realistic, values 

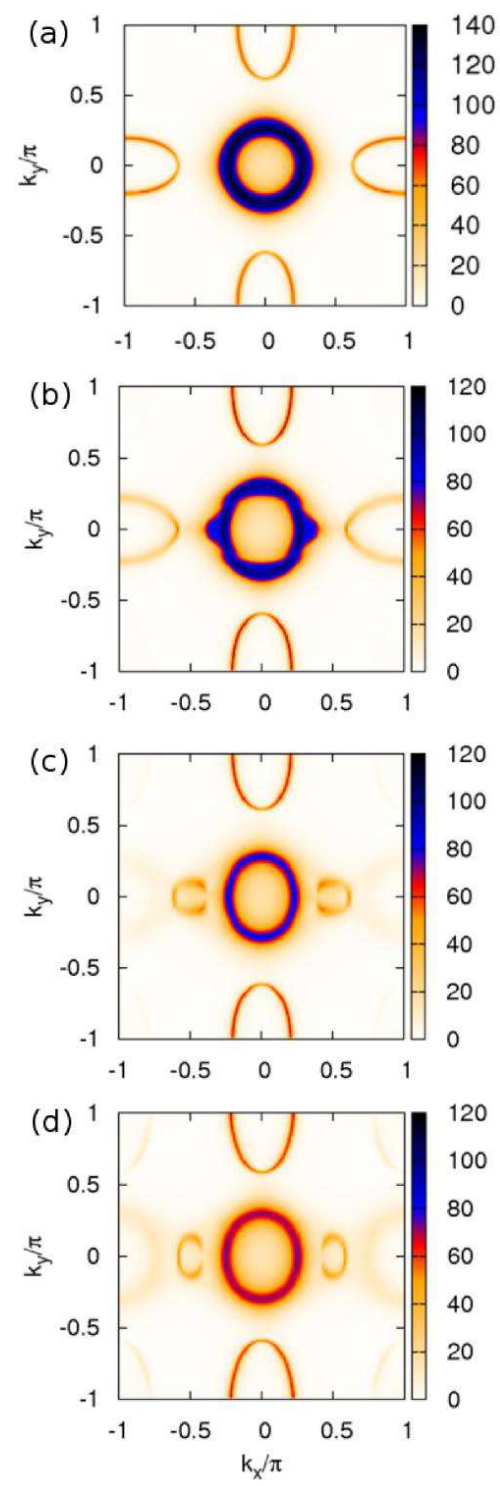

FIG. 2: Unfolded mean-field Fermi surfaces for the three-orbital model corresponding to ( $m=$ order parameter) (a) $U=0$, as reference (there are two hole pockets at $\Gamma$ but they appear merged due to the broadening used for plotting); (b) $J / U=0.33, U=0.6, m=0.2$; (c) $J / U=0.20, U=1.0, m=0.4$; and (d) $J / U=0.25, U=1.05$, $m=0.6$.

of $U$ (for more details see Ref. 31)]. The corresponding values of $J / U$ and $U$ are indicated in the figure caption. These physically acceptable Fermi surfaces from the ARPES perspective are found in the same approximate range of couplings as those selected from the $m$ /neutrons perspective, as discussed above. It has been suggested before that a reduced ordered magnetic moment goes together with realistic $A(\mathbf{k}, \omega)$ in numerical approaches like density-functional theory ${ }^{38}$ and the present mean-field scheme,, 33 and we see here that this is not accidental, but that the analysis of both neutrons and ARPES are mutually consistent over a larger parameter range, as well as for a variety of models, see Sec.IV] The four panels shown are qualitatively similar and further refinements in the so-called "physical region" (see Fig. 4) will need better tools for calculations and more accurate ARPES experiments.
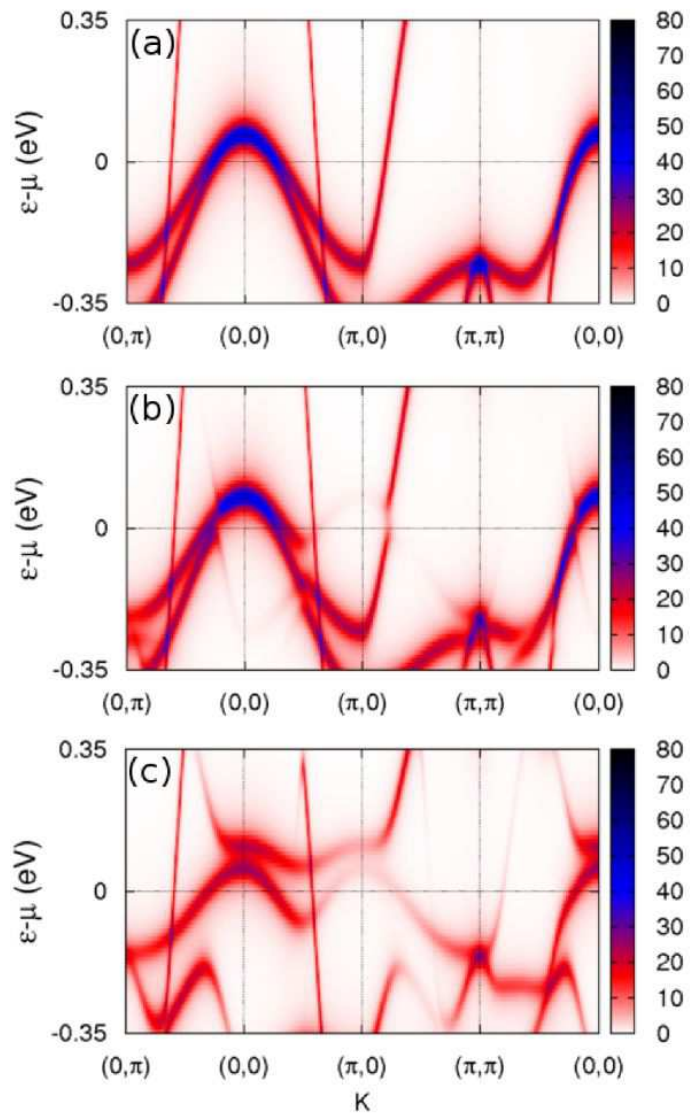

FIG. 3: Unfolded band structure mean-field results for the threeorbital model and cases (a) $U=0$, as reference; (b) $J / U=0.33$, $U=0.6, m=0.2$; (c) $J / U=0.25, U=1.05, m=0.6$. Panels (b) and (c) show a $V$-shaped pocket in between the $(0,0)$ and $(\pi, 0)$ points. The scale used (arbitrary units) is on the right of the panels.

In Fig. 3, some of the full spectral functions are shown, and compared with the $U=0$ case. The appearance of $V$-shaped features, that induce the presence of satellite pockets, is clear in these figures. These mean-field results for $A(\mathbf{k}, \omega)$ are qualitatively consistent with ARPES experiments for the pnictides that have reported similar $V$-shaped branches ${ }^{39}$

The results of Fig. 2 are in qualitative agreement with experiments, as already remarked in Ref. 33. Moreover, our comprehensive analysis of $A(\mathbf{k}, \omega)$ has shown that these features do not appear in other regions of the $U-J / U$ phase diagram. For instance, before the critical $U$ where $m$ develops from zero there are no satellite pockets, since they arise from the nonzero magnetic order and nesting effects. In the other extreme of $U$ couplings larger than those used in Fig. 2 the Hubbard model simply becomes insulating (as discussed before in Ref. 18), and there is no longer a Fermi surface. 


\section{Summary phase diagram for three orbitals}

The values of the order parameter $m$ and their comparison with neutron scattering results, and the Fermi surfaces and their comparison with photoemission experiments, lead us to the mean-field phase diagram shown in Fig. 4 , one of the main results of this effort. In this figure, the range of $U$ $J / U$ couplings compatible with neutron-ARPES experiments is labeled "physical region". This region is relatively small, providing substantial constraints on the parameters to be used in three-orbital Hubbard model investigations. If $U$ is smaller than in the physical region, then the state is not magnetic; if $U$ is larger, the state is insulating or it has a much distorted Fermi surface. If $J / U$ is smaller than in the physical region, there is no room for the small and intermediate value order parameters found in neutron scattering; if $J / U$ is larger, then $U^{\prime}$ becomes too small or negative and thus unphysical.

It is important to clarify that our mean-field approximation does not incorporate the effect of fluctuations. For this reason this type of approximations are somewhat "rigid" and it can be expected, ${ }^{29}$ although actual calculations are very difficult, that the true "physical region" may be larger than shown in Fig. 4 Thus, readers should consider the location of our "physical regions" as the center of a potentially broader area where agreement with experiments can be found. But this does not invalidate our main point: intermediate $U$ 's and intermediate $J / U$ 's are needed for agreement with available neutrons, transport, and photoemission experiments.

A final remark is with regards to the actual value of $U$ of order just $1 \mathrm{eV}$ in the physical region: this cannot be the bare $U$ but must already incorporate the influence of screening in a model where the long-range Coulomb interactions are included. It is for this reason that standard metals in general tend to have very small $U$ s when studied via Hubbard-like models, while it is known that the bare atomic values for $U$ are always of several eVs. 63

\section{RESULTS FOR FIVE-ORBITAL MODELS}

In this section, results for two five-orbital models are presented, with a similar organization as for three orbitals. The hopping amplitudes and on-site energies of a novel "Model 1 " are provided in the Appendix (using as criterion for their determination finding qualitative agreement with band calculations, as shown in Fig. 6(a)). The more accurate "Model 2 " is the model introduced in Ref. 21, where the reader can find the actual values of the parameters. These two models generate similar Fermi surfaces, but the values for the hopping amplitudes are rather different. Thus, they are useful to test whether our conclusions do or do not depend on small details. Indeed, an overall conclusion of our study is that the "physical region" is qualitatively similar for all the models analyzed in this manuscript. Both models studied in this section are at electronic density $n=6.0$ in order to address the parent compounds. ${ }^{64}$ The $\delta$-function broadening used here is 0.01 $\mathrm{eV}$.

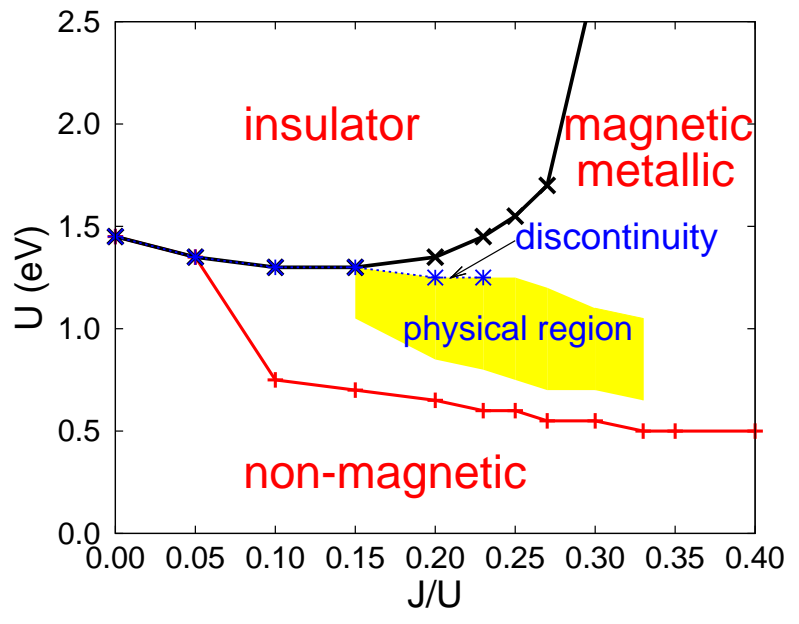

FIG. 4: Phase diagram for the three-orbital model obtained with the mean-field approximation described in the text. The "physical region" in yellow is the regime of couplings found to be compatible with neutron and photoemission experiments. The "non-magnetic" region corresponds to a regime where the state has a zero order parameter. In the "insulator" region, there is no Fermi surface and the state is insulating. The "discontinuity" label corresponds to the discontinuous jump in the order parameter shown in Fig. 1. The entire "magnetic metallic" regime could in principle have been compatible with experiments, but only in the yellow highlighted region is that $m$ is sufficiently small-intermediate in value and the Fermi surface has satellite pockets near the $\Gamma$-point hole pockets.

\section{A. Comparison with neutron scattering for Model 1}

The mean-field order parameter at wavevector $(\pi, 0)$ vs. $U$ for Model 1 is shown in Fig. 5 parametric with $J / U$. These results are in several aspects qualitatively similar to those discussed before for three orbitals in Fig. 11 but there are some differences. For instance, in this case a discontinuity in $m$ is found for all the values of $J / U$ investigated. This shows that for this model and using mean-field techniques, there is a range of values of $m$ (roughly between 1 and 2.5 depending on $J / U)$ for which there are no solutions. It turns out that neutron scattering experiments for 1111 and 122 materials have unveiled low to intermediate values of $m$, and such order parameters fit in the range where the mean-field analysis provides stable solutions. Thus, for the case of five orbitals, the comparison between the order parameter $m$ and neutron scattering simply restricts $U$ to be between the first critical value, where $m$ develops, and the second critical coupling where the discontinuity occurs. This range is larger for small $J$ than for larger values. ${ }^{25}$ However, it should be noted that the magnetization in each orbital is parallel even for small $J$ and $U$, in contrast to the state with antiparallel orbital magnetization found in Ref. 25, and in contrast to the model discussed in Sec. IVD. The small overall magnetic moment results here from weak magnetization of the individual orbitals rather than from their partial cancellation. 


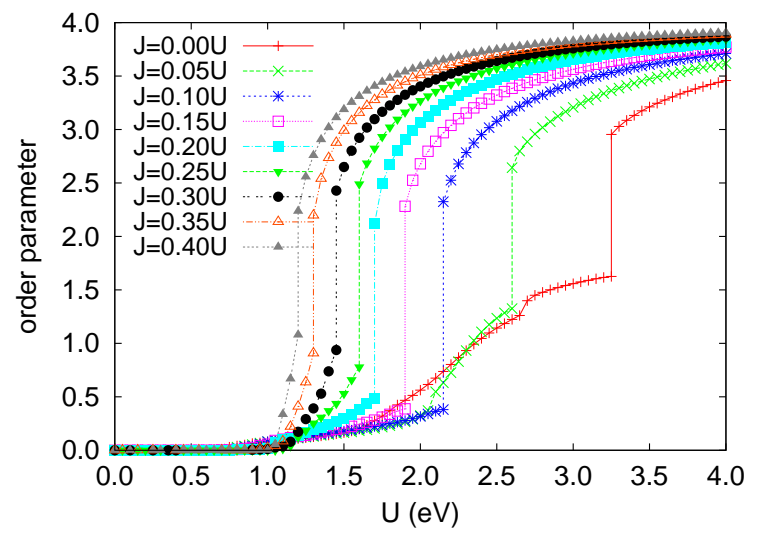

FIG. 5: Mean-field order parameter at wavevector $(\pi, 0)$ vs. $U$ (in $\mathrm{eV}$ units) for the five-orbital Model 1 discussed in the text, parametric with the values of $J / U$ indicated. Note the presence of a critical $U$ where the order parameter first develops, and a second critical $U$ where a discontinuity occurs (see also Ref.18).

\section{B. Comparison with ARPES experiments for Model 1}

The comparison between the mean-field one-particle spectral function for the five-orbital models and ARPES experiments introduces more severe restrictions on the values of $J / U$ and $U$ than those discussed before in the neutron scattering context. Figure 6 shows the Fermi surfaces of Model 1 for special values of $J / U$ and $U$ in a region compatible with ARPES. Qualitatively, these results resemble those of the three-orbital model: there are remnants of the original hole and electron pockets of the non-interacting limit and, in addition, there are satellite features near the $\Gamma$-point (and also near the original non-interacting electron pocket at $(\pi, 0)$ ).

Figure 7 shows the actual bands for the five-orbital Model 1 for the non-interacting case and one example of a set of couplings with satellite pockets created by the magnetic order. While qualitatively similar to the results for three orbitals (Fig. 3) there are some interesting differences: here the $V$ shaped features that originate the satellite pockets arise from a combination of two bands while for three orbitals they emerge from the bending of a single band (see also Ref. 33). However, the level of accuracy of the ARPES experiments is not sufficient to distinguish between these two cases, plus there is still room to further refine the hopping amplitudes of the models used here to adjust for finer details of the ARPES experiments.

Even in regions of parameter space where the ground state is magnetic and metallic, and the order parameter is in the range of neutron's experiments, the Fermi surface may still be qualitatively different from that observed in ARPES. As an example, consider the case shown in Fig. 8 corresponding to $J / U=0.10$. These mean-field results for $A(\mathbf{k}, \omega)$ are clearly different from those shown before in Fig. 6, and regions where this type of discrepancies are found are removed from the "physical region" for the model.
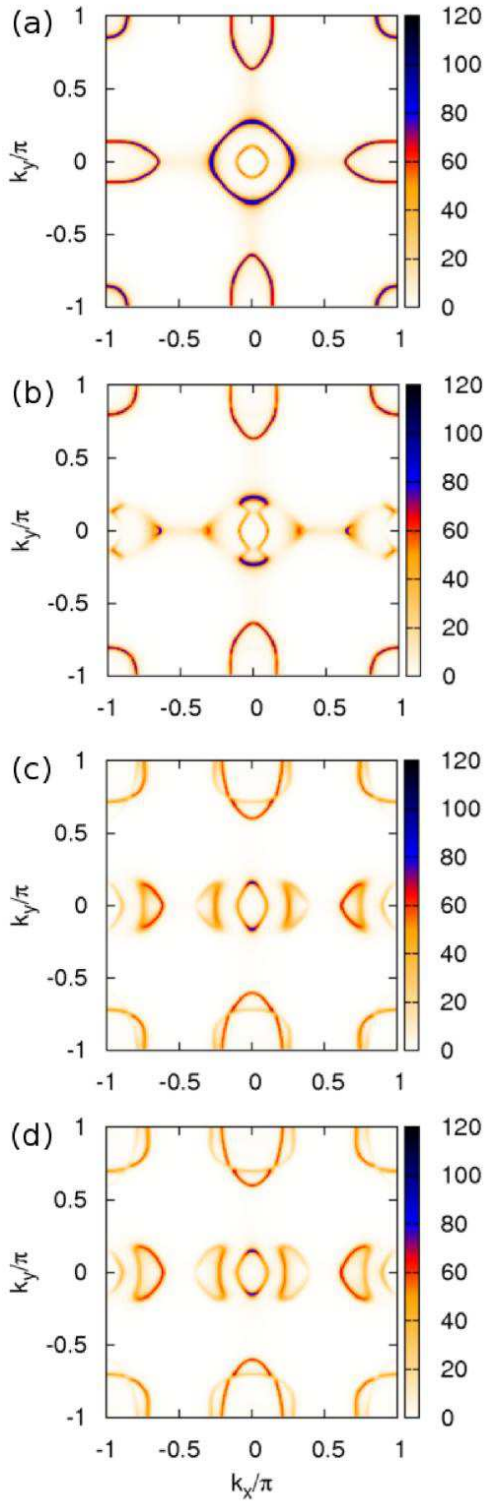

FIG. 6: Unfolded Fermi surfaces of the five-orbital Model 1 in the mean-field approximation for the cases (a) $U=J=0$, as reference; (b) $J / U=0.23, U=1.25, m=0.2$; (c) $J / U=0.28, U=1.45$, $m=0.7$; and (d) $J / U=0.30, U=1.4, m=0.7$. As in the case of three orbitals (Fig.2) and as in ARPES experiments, the results show distorted $\Gamma$-point hole pockets and satellite features next to them (and also next to the electron pockets at $(\pi, 0)$ ).

\section{Summary phase diagram for the five orbitals Model 1}

Similarly as for three orbitals, here a summary phase diagram is provided for the five-orbital Model 1 in Fig. 9. The labeling convention is the same as for three orbitals in Fig. 4. Using the information about neutron scattering and order parameters restricts $U$ and $J / U$ simply to be between the nonmagnetic region and the discontinuity line. From these perspectives alone the "physical region" would be much larger 


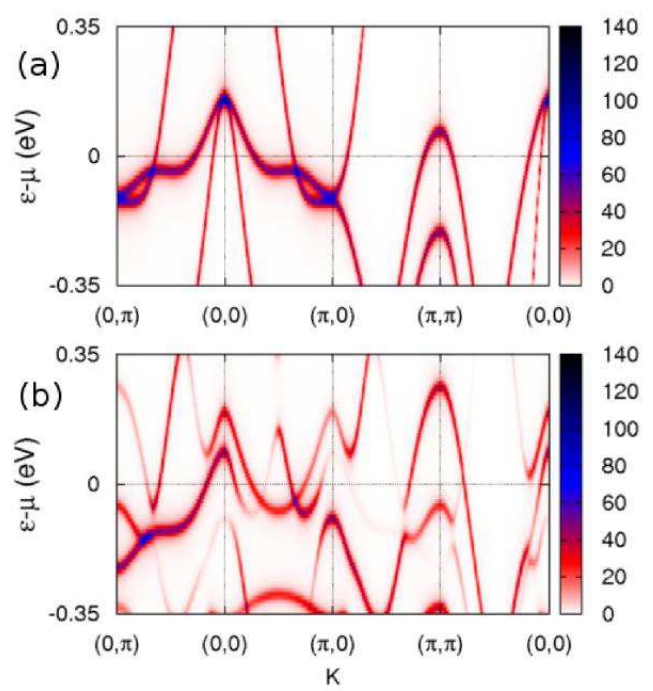

FIG. 7: Unfolded band structure mean-field results for the fiveorbital Model 1 and cases (a) $U=J=0$, as reference, and (b) $J / U=0.28, U=1.45, m=0.7$, illustrating in (b) the origin of the satellite pockets shown in Fig. 6 The scale used (arbitrary units) is on the right.

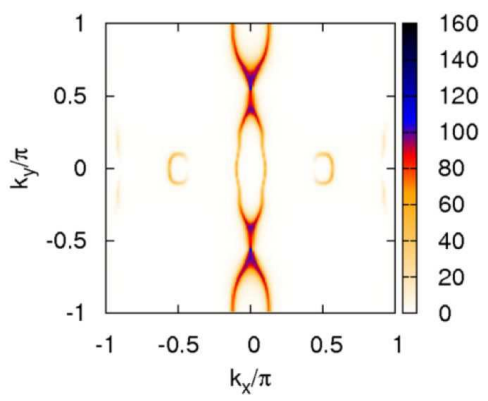

FIG. 8: Fermi surface of the five-orbital Model 1 in the mean-field approximation for the case $J / U=0.10, U=2.1$, and $m=0.36$. The lack of qualitative similarity with ARPES experiments shows that these couplings are not physically relevant to describe the pnictides.

than for the three orbitals case. However, considering the Fermi surface shape and its comparison with ARPES introduces more severe constraints, basically excluding the small $J / U$ regime below 0.15 . As a consequence, the final "physical region" ends up being similar to that obtained with the threeorbital model. As explained in Sec. III.C, note also that fluctuations beyond our mean-field approximation are expected to render the "physical region" actually larger than displayed in Fig. 9

\section{Comparison with neutron scattering for Model 2}

The mean-field order parameter at wavevector $(\pi, 0)$ vs. $U$ is shown for Model 2 in Fig. 10, parametric with $J / U$. Com-

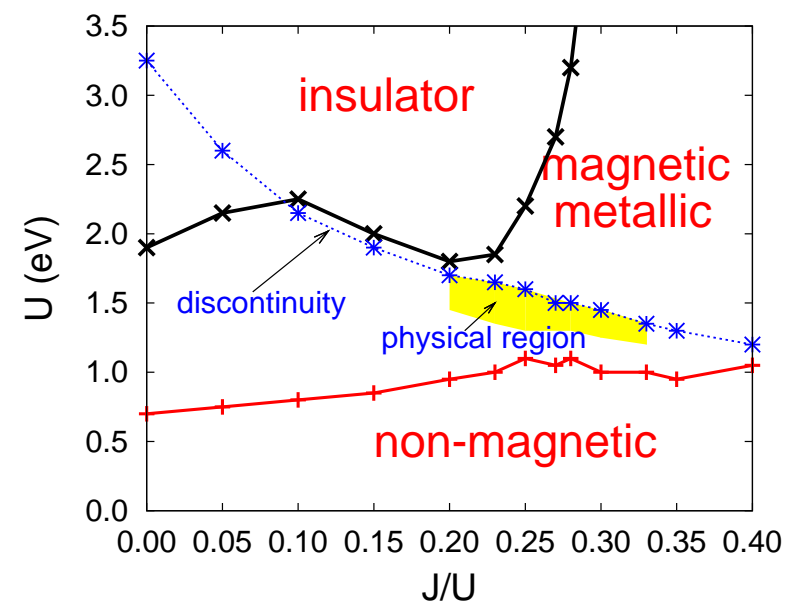

FIG. 9: Phase diagram for the five-orbital Model 1 obtained with the mean-field approximation. As in Fig. 4 the "physical region" in yellow is the regime of couplings found to be compatible with neutron and photoemission experiments. The rest of the notation and details were already explained in Fig. 4

pared with the results for Model 1 in Fig. 5, note that now the discontinuity in the order parameter occurs only at small $J / U$. In this respect, the results for Model 1 are similar to those recently reported for a similar model, ${ }^{25}$ where small $\mathrm{J} / U$ and intermediate $U$ were emphasized (see also Ref. 18). This similarity persists in the microscopic details of the state realized at small $J$ : The values for the magnetization of different orbitals can here have a different sign, i.e., the intermediatespin state with antiparallel orbital magnetization discussed in Ref. 25 is stabilized. While neutron scattering can only detect the overall moment and is not expected to distinguish between this scenario and the one with parallel magnetization found in other models, the precise microscopic nature of the state realized in iron pnictide compounds remains to be clarified.

\section{E. Comparison with ARPES experiments for Model 2}

Similarly as presented for Model 1 and for the three-orbital model, Figure 11 shows the Fermi surfaces for special values of $J / U$ and $U$ in a region found to be qualitatively compatible with ARPES. These Fermi surfaces show remnants of the non-interacting Fermi surfaces, plus satellite extra features near the $\Gamma$ - and $(\pi, 0))$-points. Note that the character of these satellites, namely whether they are electron or hole pockets, depends on details and our focus has only been on the existence of extra features in the correct location as compared with ARPES. Figure 12] shows the bands for the five-orbital Model 2 , both for the non-interacting case and for one example from the set of couplings used in Fig. 11 Most of the comments already made for the band structure of Model 1 with regards to the $V$-shaped features apply to Model 2 as well. However, it is interesting to notice that the satellite pockets are mainly electron-like for Model 1 and hole-like for Model 2. 


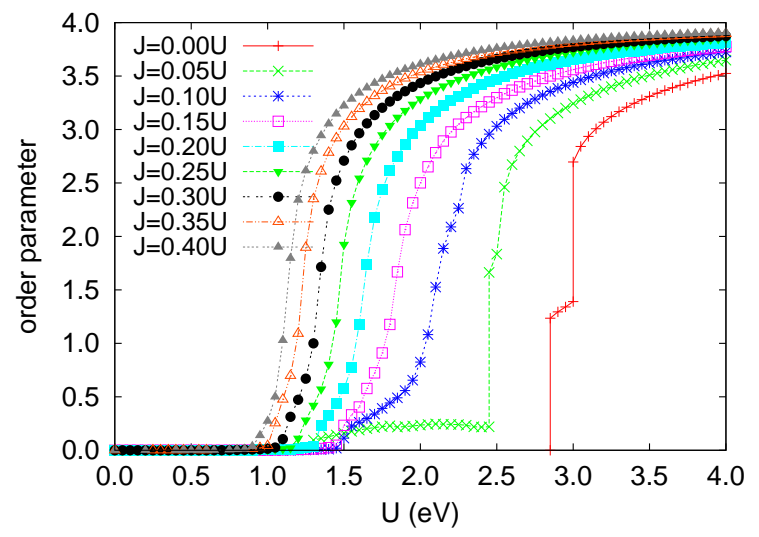

FIG. 10: Mean-field order parameter at wavevector $(\pi, 0)$ vs. $U$ (in $\mathrm{eV}$ units) for the five-orbital Model 2, parametric with the values of $J / U$ indicated. Note that compared with Fig. 5 the discontinuity in the order parameter now only occurs at small $J / U$.

\section{F. Summary phase diagram for the five orbitals Model 2}

As for the other models considered in this effort, in Fig. 13]a summary phase diagram is provided for the five-orbital Model 2. The labeling convention is the same as in Figs. 4 and 9 , as well as the procedure to establish the so-called "physical region". The approximate location of this region with regards to $U$ and $J / U$ is similar to that reported in Figs. 4 and 9 showing again that our conclusions do not strongly depend on details. The physical region in Fig. 13 is narrow along the $U$ axis direction because $m$ changes rapidly with increasing $U$, at small $m$. But, as discussed in Sec. III.C, fluctuations beyond mean-field approximations are expected to expand the size of the physical regions of the various models.

\section{RPA PAIRING SYMMETRIES ANALYSIS}

After having established a regime of couplings $U$ and $J / U$ where the mean-field approximation to the multiorbital Hubbard model gives a qualitative agreement with neutron and ARPES experiments, and also with the metallic nature of the undoped compounds, it is interesting to investigate what kind of pairing tendencies are observed in those regions of parameter space. Unfortunately, the number of many-body tools available to carry out such investigation is very small. While for the two-orbital model it is possible to carry out Lanczos studies on small clusters $\underline{\underline{13}}$ to at least analyze the quantum numbers of the ground state with two extra particles, for three orbitals or more this calculation is no longer practical. There are also no sign-problem-free Monte Carlo techniques available for these complex calculations. Thus, here the Random Phase Approximation will be used. Experience with the cuprates indicates that this method did capture the relevance of $d$-wave pairing in that context and for this reason it will be used here as well, although with the caveat that the method
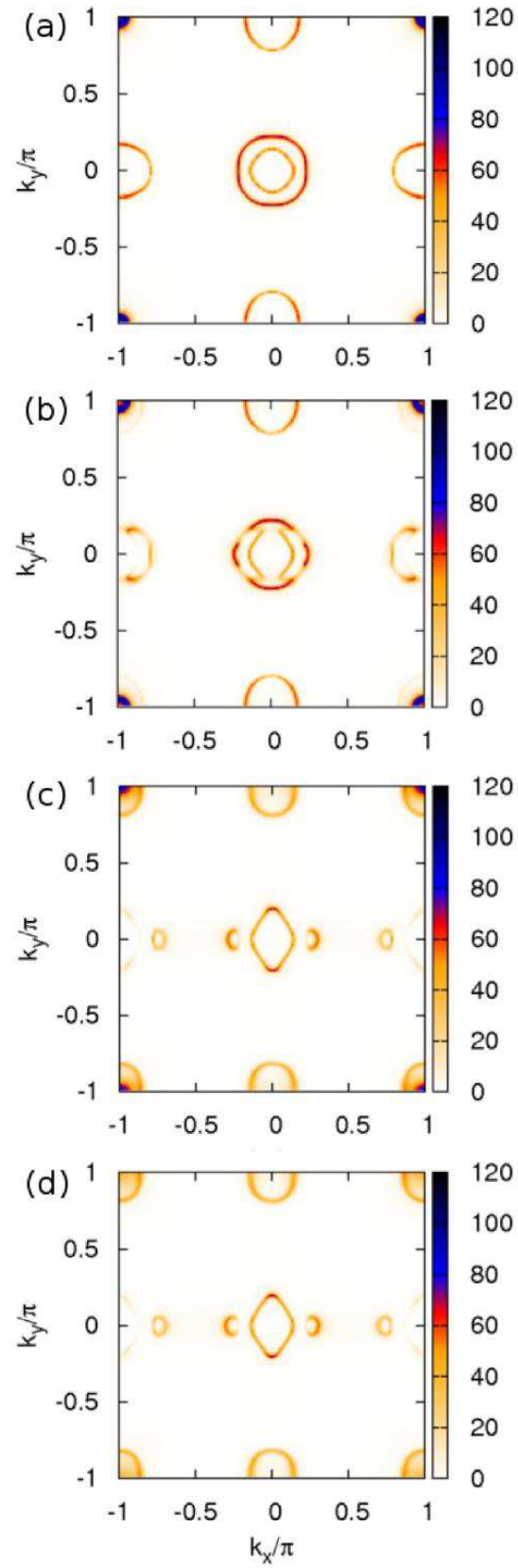

FIG. 11: Unfolded Fermi surfaces of the five-orbital Model 2 in the mean-field approximation at $n=6.0$, for (a) $U=J=0$, as reference; (b) $J / U=0.20, U=1.35, m=0.2$; (c) $J / U=0.25, U=1.35$, $m=0.5$; and (d) $J / U=0.30, U=1.25, m=0.6$. As in the previous cases (Figs. 2] and 6) and as in ARPES experiments, the results show distorted $\Gamma$-point hole pockets and satellite features next to them (and also next to the electron pockets at $(\pi, 0))$.

is qualitative at best. RPA has been recently applied to the five-orbital Model 2 considered here 21 and the dominance of $A_{1 g}$ states was unveiled at small $J / U$, with $B_{1 g}$ a close competitor. In our investigations shown below, in the "realistic" regime of couplings the RPA dominant pairing tendency always has nodes or quasi-nodes, an interesting result in view 

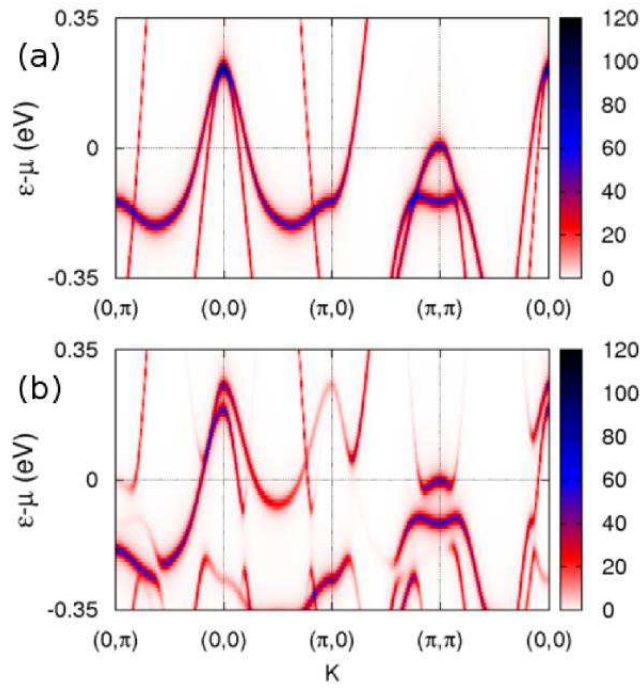

FIG. 12: Unfolded band structure mean-field results for the fiveorbital Model 2 and cases (a) $U=J=0$, as reference, and (b) $J / U=0.25, U=1.35, m=0.5$, illustrating in (b) the satellite pockets shown in Fig.11 The scale used (arbitrary units) is on the right. Note the similarity of these results with those of Fig. 7

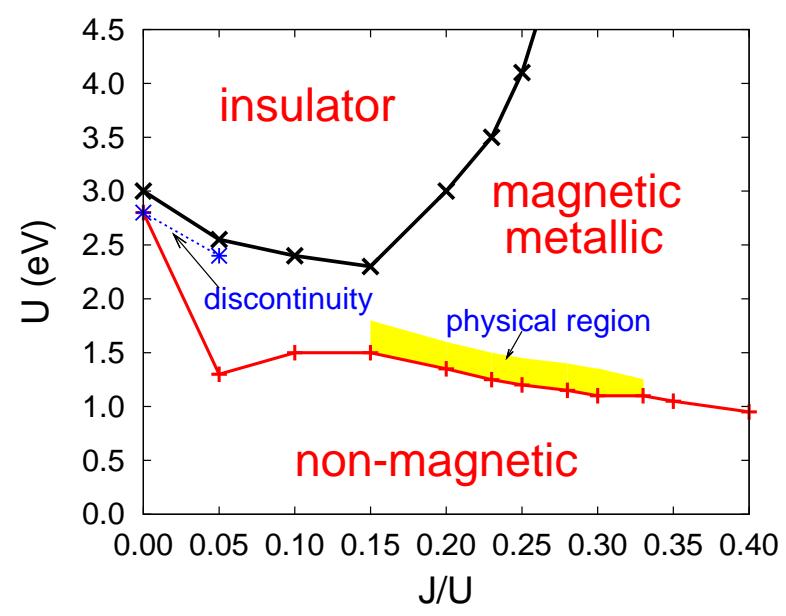

FIG. 13: Phase diagram for the five-orbital Model 2 obtained with the mean-field approximation. As in Figs. 4 and 9 the "physical region" in yellow is the regime of couplings found to be compatible with neutron and photoemission experiments. The rest of the notation and details were already explained in Fig. 4

of the current experimental controversy between ARPES and other techniques with regards to the nodal structure of the superconducting state. It is also important to remark that other irreducible representations are closely competing with those that dominate, and small changes in parameters alter their relative dominance.

\section{A. Random Phase Approximation Formalism}

For completeness, here the RPA approximation will be briefly reviewed, with emphasis on some technical aspects. This section follows the formalism already described in Refs. 21 and 65. Assuming that spin fluctuations (excitations in the paramagnetic state above the critical temperature, also called paramagnons) are responsible for the pairing mechanism present in the iron-pnictides, the RPA method will be used to study these fluctuations (caused by itinerant carriers) beyond the mean-field level. In linear response theory, prior to including the many-body interactions, these spin-wave-like excitations are obtained from the non-interacting Lindhard function (here defined for a multi-orbital model)

$$
\chi_{l_{1}, l_{2}, l_{3}, l_{4}}^{0}(\mathbf{q})=\sum_{\mathbf{k}} G_{l_{1}, l_{3}}(\mathbf{k}+\mathbf{q}) G_{l_{4}, l_{2}}(\mathbf{k}),
$$

where each of the four indices needed to characterize the Lindhard function takes the values $l_{i}=1, \ldots, n_{o}(i=1, \ldots, 4)$, with $n_{o}$ the total number of orbitals being considered (here, $n_{o}=3$ or $n_{o}=5$ ). The non-interacting Green's function, which describes the propagation of the elementary excitations present in the non-interacting model, can be written as

$$
G_{l_{1}, l_{3}}(\mathbf{k}, \omega)=\sum_{\mu} \frac{\left\langle l_{1} \mid \mu \mathbf{k}\right\rangle\left\langle\mu \mathbf{k} \mid l_{3}\right\rangle}{\omega-E_{\mu}(\mathbf{k})}
$$

where $b_{\mu}^{l_{1}}(\mathbf{k})=\left\langle l_{1} \mid \mu \mathbf{k}\right\rangle$ projects the tight-binding state $|\mu \mathbf{k}\rangle$ into the orbital $\left|l_{1}\right\rangle$, where $\mu$ labels which one of the $2 n_{o}$ tightbinding bands (in the extended BZ, with one iron per unit cell) the tight-binding state belongs to (with energy $E_{\mu}(\mathbf{k})$ ). Introducing many-body interactions in the model, the Lindhard function gives origin to (distinct) spin and charge susceptibilities, which, calculated at the RPA level through a Dyson equation, can be expressed as (using matrix equations),

$$
\begin{aligned}
& \hat{\chi}_{s}(\mathbf{q})=\frac{\hat{\chi}^{0}(\mathbf{q})}{1-\hat{U}^{s} \hat{\chi}^{0}(\mathbf{q})}, \\
& \hat{\chi}_{c}(\mathbf{q})=\frac{\hat{\chi}^{0}(\mathbf{q})}{1+\hat{U}^{c} \hat{\chi}^{0}(\mathbf{q})},
\end{aligned}
$$

where expressions for the non-zero matrix elements of $\hat{U}^{s}$ and $\hat{U}^{c}$, in terms of the many-body interactions, can be found in Refs. 21 and 65. The magnetic susceptibility (both RPA and bare) to be shown below in, e.g., Fig. 14 is given by

$$
\chi_{s}(\mathbf{q})=\frac{1}{2} \sum_{l_{1}, l_{2}}\left(\hat{\chi}_{s}\right)(\mathbf{q})_{l_{1}, l_{1}}^{l_{2}, l_{2}} .
$$

These RPA susceptibilities are used to construct a spinsinglet pairing interaction describing the exchange of charge (orbital) and spin fluctuations, resulting in an effective electron-electron interaction: ${ }^{66}$

$$
\hat{V}^{s}(\mathbf{q})=\frac{3}{2} \hat{U}^{s} \hat{\chi}_{s}(\mathbf{q}) \hat{U}^{s}-\frac{1}{2} \hat{U}^{c} \hat{\chi}_{c}(\mathbf{q}) \hat{U}^{c}+\frac{1}{2}\left(\hat{U}^{s}+\hat{U}^{c}\right) .
$$


Assuming that the dominant scattering occurs close to the Fermi surface, one can calculate the scattering amplitude of a Cooper pair between two points at the Fermi surface $\left[(\mathbf{k},-\mathbf{k}) \rightarrow\left(\mathbf{k}^{\prime},-\mathbf{k}^{\prime}\right)\right]$, where the momenta $\mathbf{k}$ and $\mathbf{k}^{\prime}$ are restricted to the Fermi surface pockets $i$ and $j$, which span the existing pockets for the chemical potential used. To indicate this restriction a subscript $i$ (or $j$ ) is added to the bands $\mu$ and $v$ in question, and the (symmetrized) interaction vertex becomes:

$$
\begin{aligned}
\Gamma_{i j}\left(\mathbf{k}, \mathbf{k}^{\prime}\right) & =\sum_{l_{1}, l_{2}, l_{3}, l_{4}} b_{\mu_{i}}^{l_{2}, *}(-\mathbf{k}) b_{\mu_{i}}^{l_{1}, *}(\mathbf{k}) \operatorname{Re}\left[V_{l_{1}, l_{2}}^{l_{3}, l_{4}}\left(\mathbf{k}, \mathbf{k}^{\prime}\right)\right] \\
& \times b_{v_{j}}^{l_{3}}\left(\mathbf{k}^{\prime}\right) b_{v_{j}}^{l_{4}}\left(-\mathbf{k}^{\prime}\right),
\end{aligned}
$$

which, after plugging into the linearized Eliashberg equation, results in a dimensionless pairing strength functional: ${ }^{67,68}$

$$
\lambda[g(\mathbf{k})]=-\frac{\sum_{i j} \oint_{C_{i}} \frac{d k_{\|}}{v_{F}(\mathbf{k})} \oint_{C_{j}} \frac{d k_{\|}^{\prime}}{v_{F}\left(\mathbf{k}^{\prime}\right)} g(\mathbf{k}) \Gamma_{i j}\left(\mathbf{k}, \mathbf{k}^{\prime}\right) g\left(\mathbf{k}^{\prime}\right)}{4 \pi^{2} \sum_{i} \oint_{C_{i}} \frac{d k_{\|}}{v_{F}(\mathbf{k})}[g(\mathbf{k})]^{2}},
$$

where the superconducting gap $\Delta(\mathbf{k})$ (in reciprocal space) was separated into an amplitude $\Delta$ and a function $g(\mathbf{k})$, which should have the symmetry of one of the irreducible representations of the corresponding point group (in the case of the pnictides, the $D_{4 h}$ group is considered, with representations $A_{1 g}, A_{2 g}, B_{1 g}, B_{2 g}$, and $E_{g}$ ). The stationary condition leads to an eigenvalue problem defined over the Fermi surface:

$$
-\sum_{j} \oint_{C_{j}} d k_{\|}^{\prime} \frac{1}{4 \pi^{2} v_{F}\left(\mathbf{k}^{\prime}\right)} \Gamma_{i j}\left(\mathbf{k}, \mathbf{k}^{\prime}\right) g_{j}\left(\mathbf{k}^{\prime}\right)=\lambda g_{i}(\mathbf{k}),
$$

which is an eigenvalue problem involving a matrix $\left[\Gamma\left(\mathbf{k}, \mathbf{k}^{\prime}\right)\right]$, and where $g_{i}(\mathbf{k})$ is the value of the gap function for a point $\mathbf{k}$ at the pocket $i$, and $\lambda$ is the associated eigenvalue. The highest eigenvalue (normalized to 1) indicates what gap function will have the highest critical temperature. The dimension of the matrices to be diagonalized will be determined by the number of $\mathbf{k}$ points taken along the Fermi surface. For the calculations shown here, it was observed that considering approximately 200 points along the Fermi surface provides a good convergence. All calculations were done at temperature $T=0.02 \mathrm{eV}$, and an imaginary part $\eta=10^{-5}$ was used to regularize the Green's functions. All the sums over the BZ were done with uniform $64 \times 64$ meshes (calculations with a $128 \times 128$ mesh yielded qualitatively the same results).

\section{B. RPA Pairing Symmetries for the Five-Orbital Model 1}

The RPA analysis of the "realistic" $U$ and $J / U$ regime will start here with the five-orbital Model 1. In Fig. 14, the RPA results for the magnetic susceptibility $\chi_{S}$ are shown for two values of $J / U$ (and slightly different $U$ ). The case $J / U=0.15$ is close but outside the "physical region" of Fig. 9, while $J / U=0.28$ is clearly inside that region. However, with regards to $\chi_{S}$, the figure shows that there are no substantial changes in the magnetic response for these two $J / U \mathrm{~s}$ : an approximately flat bare $\chi_{S}$ becomes a RPA $\chi_{S}$ with a sharp peak at the correct wavevector $(\pi, 0)$.

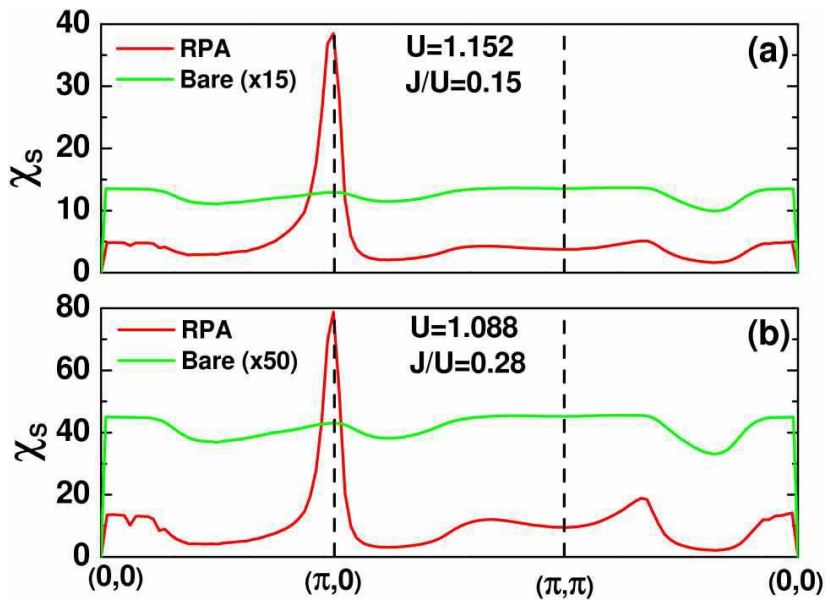

FIG. 14: Bare and RPA magnetic susceptibilities vs. BZ wavevector, at the values of $U$ and $J / U$ indicated, for the five-orbital Model 1 .

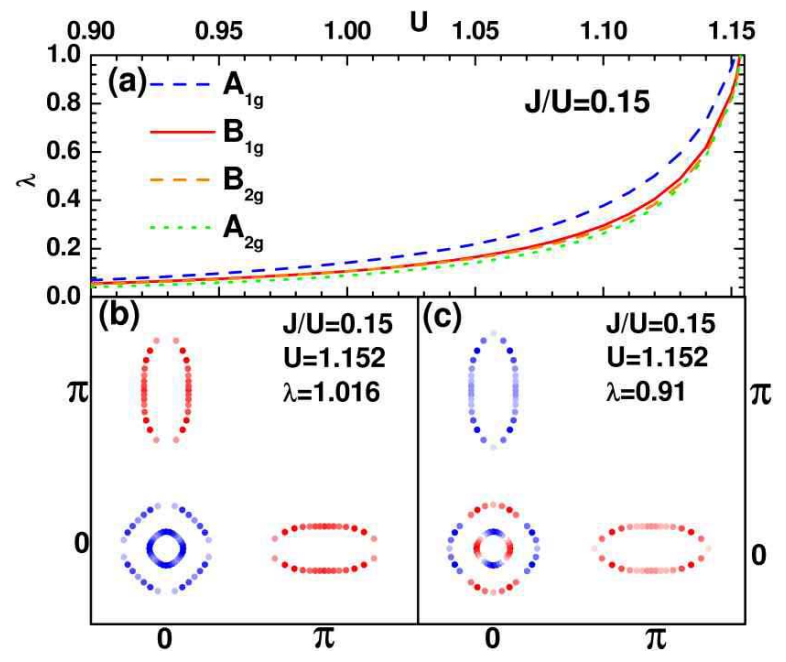

FIG. 15: (a) RPA pairing eigenvalues vs. $U$, at $J / U=0.15$, for the five-orbital Model 1. The symmetries of each eigenvalue are indicated. (b) Dominant $A_{1 g}$ gap function with a similar color convention as in Ref. 21 (blue and red denoting different signs). (c) Subdominant state belonging to the $B_{1 g}$ representation.

The RPA pairing eigenvalues $\lambda$ are shown in Fig. 15 (a) as a function of $U$, at a constant $J / U=0.15$. Shown in (a) are the four first eigenvalues, that happened to have the four different symmetries that are indicated (as opposed to repeating some symmetries). The dominant one is $A_{1 g}$, in agreement with Ref. 21 using the five-orbital Model 2. The actual pairing function for this dominant eigenvalue is shown in Fig. 15(b). This is qualitatively of the form of the well-known $s^{+-}$pairing, with different signs between the hole and electron pockets. However, the actual gap values are not uniform along each pocket. Actually, all the pockets present some narrow regions where the gap functions nearly vanish, a feature quite reminiscent of nodes (dubbed here quasi-nodes 69 ). Overall, these results, and those presented below for Model 2 and for 
the three-orbital model, are compatible with the recent observations in Refs. 22, 65 that pockets at $(\pi, \pi)$ suppress nodes in the $A_{1 g}$ sector while the absence of those pockets produce a nodal $A_{1 g}$ (note that in some of our results here and below, the superconducting order parameter at $(\pi, \pi)$ is weak and difficult to see).

In Fig. 15 (c), the pairing function of the subdominant eigenvalue is shown. By mere inspection it is clear that it belongs to the $B_{1 g}$ sector, in qualitative agreement with Ref. 21 that also identified this symmetry as the first competitor to $A_{1 g}$. Thus, for $J / U=0.15$ our results are similar to those previously reported $^{21}$ and have the value of providing a test of our procedure. However, Fig. 15 (a) contains extra information not discussed before: it also shows that two other eigenvalues are nearly degenerate with $B_{1 g}$ and in view of the approximate nature of the calculation they should also be considered as important competitors. These extra competitors belong to the $B_{2 g}$ and $A_{2 g}$ sectors. In particular the $B_{2 g}$ is compatible with the Lanczos results found for the two-orbital model in a similar "intermediate $U$ " region of parameter space. ${ }^{13}$

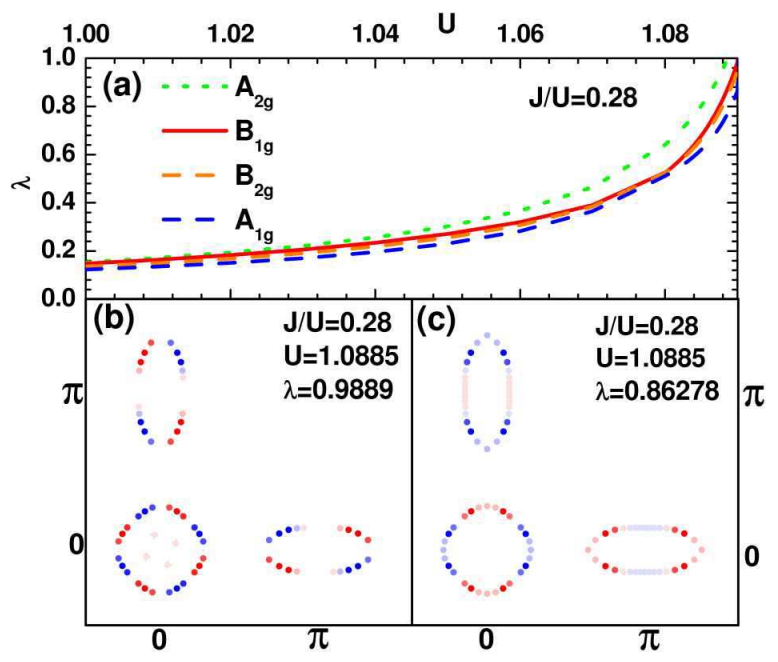

FIG. 16: (a) RPA pairing eigenvalues vs. $U$, at $J / U=0.28$, for the five-orbital Model 1. The symmetries of each eigenvalue are indicated. (b) Dominant $A_{2 g}$ gap function with a similar color convention as in Ref. 21 (blue and red denoting different signs). (c) Subdominant state belonging to the $B_{1 g}$ representation.

Figure 16 provides novel interesting information. Switching now to $J / U=0.28$, i.e. inside the $U-J / U$ region considered realistic based on neutron and ARPES experiments, the dominant eigenvalue now belongs to the $A_{2 g}$ irreducible representation (with a gap function shown in (b)). $B_{1 g}$ (see (c)) is still the subdominant tendency, but it is nearly degenerate with $B_{2 g}$. For this value of $J / U$ the $A_{1 g}$ channel is 4 th in the relative order. Thus, a relatively small variation in $J / U$ induces a qualitatively drastic rearrangement of pairing eigenvalues. However, all of them have nodes (or quasi-nodes as in the case of $A_{1 g}$ ). Thus, it appears unavoidable to conclude that nodal superconductivity should be present in the pnictides, at least within pairing tendencies that rely on electronic mechanisms (and within the RPA and mean-field approximations).
The presence of pairing states with nodes also extends to the regime of electron doping. For instance, for density $n=$ 6.125 our RPA studies (not shown) indicate the dominance of a $B_{1 g}$ state for $J / U=0.28$ and 0.35 , with an $A_{1 g}$ state with nodes being the subdominant pairing. While small changes alter the relative balance between the many competing states, all of those states, including the $A_{1 g}$, present a nodal (or quasinodal) structure at the RPA level used here.

It is worth discussing these results in a historical context. In previous RPA studies of one-orbital Hubbard models in the spin-singlet channel, it was observed a dominance of the wellknown $d_{x^{2}-y^{2}}$-wave state over other channels. ${ }^{67}$ Calculations in the one-orbital context did not report the presence of other pairing states so close to the dominant one as shown here and in Ref. 21. In fact, the only competitors to the $d$-wave state that have been identified for the case of one orbital are spintriplet $s$-wave odd-frequency states (not studied in our present investigations due to their spin-triplet nature). The appearance of odd-frequency states as competitors was analyzed in studies on square lattices, ${ }^{70}$ as well as on triangular lattices. ${ }^{71}$ But among the spin-singlet states, the dominance of $d$-wave was clear for one orbital. Thus, finding in the multiorbital models so many spin-singlet even-frequency states competing with the dominant ones is surprising and merits further work. These results suggest that within the pnictide family different pairing channels may be stable in different pnictide compounds since small changes in parameters, such as caused by chemical doping or pressure, may lead to changes in the dominant pairing tendency.

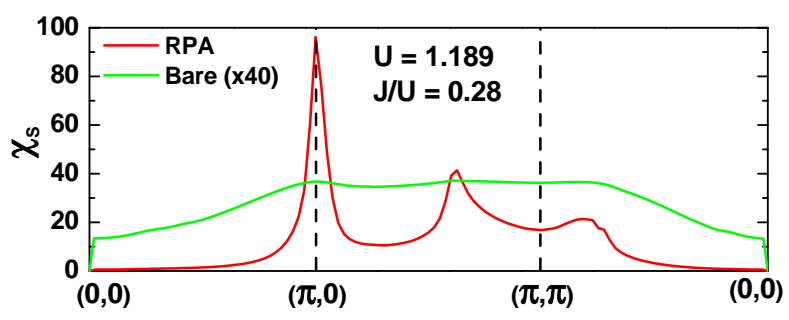

FIG. 17: Bare and RPA magnetic susceptibilities vs. BZ wavevector, at the values of $U$ and $J / U$ indicated, for the five-orbital Model 2.

\section{RPA Pairing Symmetries for the Five-Orbital Model 2}

For completeness, the RPA analysis for Model 2 is here included. Model 2 was already studied in Ref. 21, but here the focus will be on the "realistic" regime of $U$ and $J / U$. In Fig. 17 the RPA results for the magnetic susceptibility $\chi_{S}$ are shown for representative values of $U$ and $J / U$. In this regime, there is a clearly dominant peak at wavevector $(\pi, 0)$, although there are subdominant peaks at other momenta as well. The results at other value of $U$ and $J / U$ in the vicinity of the one shown are similar.

The corresponding RPA pairing eigenvalues $\lambda$ are shown in Fig. 18 (a) as a function of $U$, at a constant $J / U=0.28$. Following a similar organization as in the case of Model 1 , shown 


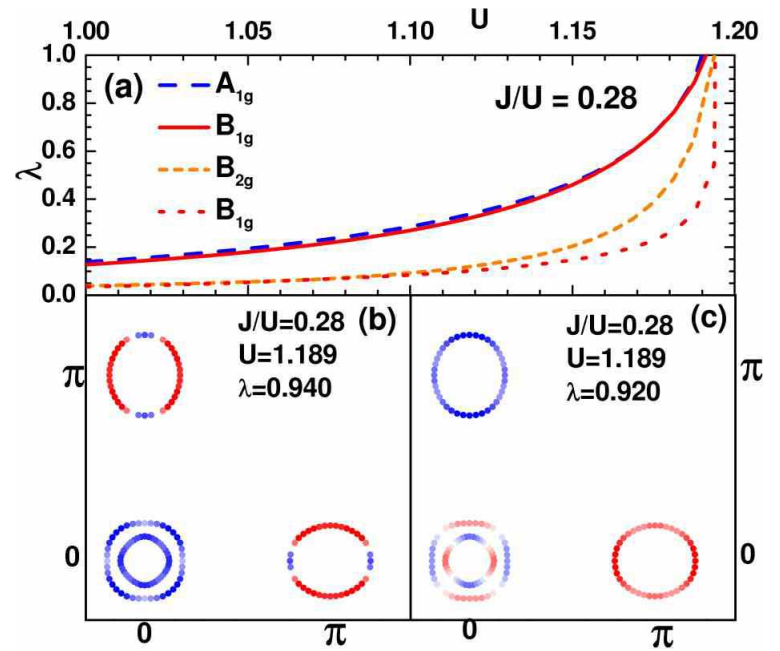

FIG. 18: (a) RPA pairing eigenvalues vs. $U$, at $J / U=0.28$, for the five-orbital Model 2. The symmetries of each eigenvalue are indicated. (b) Dominant $A_{1 g}$ gap function with a similar color convention as in Ref. 21 (blue and red denoting different signs). (c) Subdominant state belonging to the $B_{1 g}$ representation.

in (a) are the four first eigenvalues. The dominant one is $A_{1 g}$ with nodes, in agreement with Refs. 21,22. The pairing function for this dominant eigenvalue is shown in Fig.18](b): it has different signs between the hole and electron pockets, and the actual gap values are not uniform along each pocket, actually presenting nodes. In Fig. 18 (c), the pairing function of the subdominant eigenvalue is shown. It belongs to the $B_{1 g}$ sector, as found in Ref. 21. The importance of our results is that using exactly the same formalism our approach does properly reproduce previous results with regards to the dominance of the $A_{1 g}$ sector for Model 2, while Model 1 (with a very similar Fermi surface) has pairing in other channels. This highlights again that very small changes in parameters can dramatically alter the pairing instability channel.

\section{RPA Pairing Symmetries for the Three-Orbital Model}

To complete the RPA analysis, now the three-orbital model will be considered. The results reported below will not be as clear as in the previous case of five orbitals, but these results are presented here anyway to alert the reader of the subtleties associated with RPA approximations. In spite of the difficulties to be shown below, the pairing states that dominate still have nodes. Thus, the general conclusion that nodal superconductivity tends to be favored in Hubbard models remains the same, at least within the approximations used in our effort.

Figure 19 shows the RPA magnetic susceptibility for two cases of interest, $J / U=0.15$ and 0.30 , within the "physical region" of Fig. 4 These results already illustrate the main problem found here: although there is a small peak at wavevector $(\pi, 0)$, there are other larger peaks at wavevectors closer to $(0,0)$. Thus, the results to be shown for the pairing tendencies are in a magnetic background that does not cor-
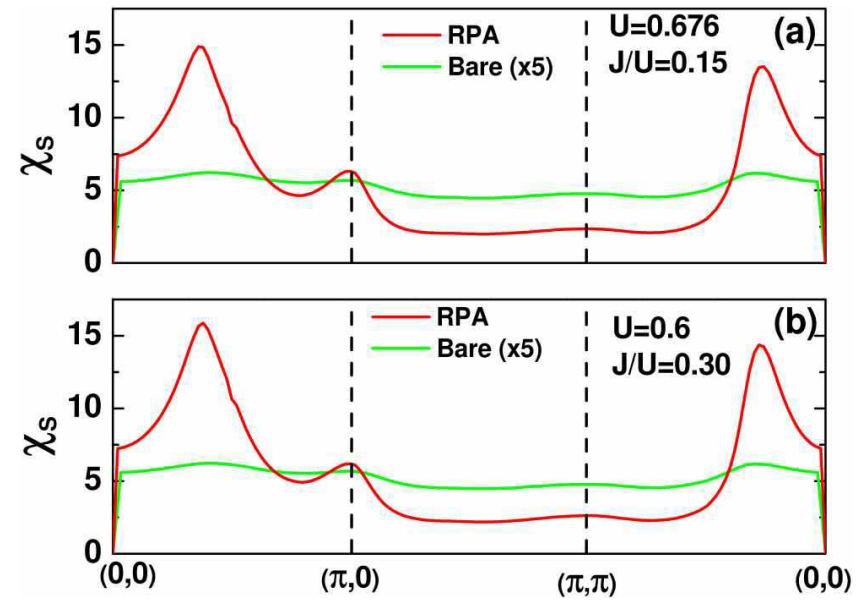

FIG. 19: Bare and RPA magnetic susceptibility vs. BZ wavevector, at the values of $U$ and $J / U$ indicated, for the three-orbital model. Note that in addition to a small peak at $(\pi, 0)$, there are larger peaks at other wavevectors.

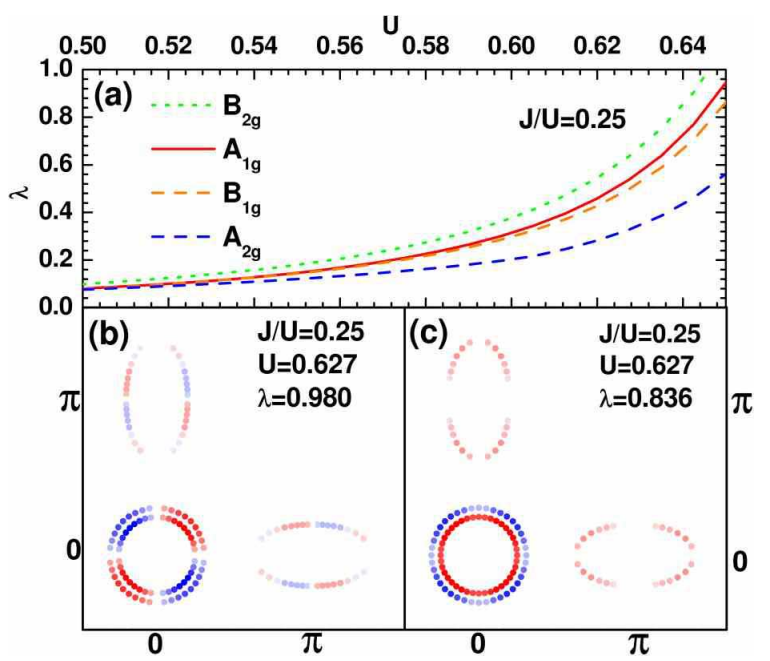

FIG. 20: (a) RPA pairing eigenvalues vs. $U$, at $J / U=0.25$, for the three-orbital model. The symmetries of each eigenvalue are indicated. (b) Dominant $B_{2 g}$ gap function with a similar color convention as in Ref. 21 (blue and red denoting different signs). (c) Subdominant state belonging to the $A_{1 g}$ representation.

respond to that of the pnictides experiments, but it contains states with a variety of wavevectors.

Figure 20 (a) displays the $U$ dependence of the pairing eigenvalues at $J / U=0.25$, inside the "physical region" for three orbitals. Note that in this case it is the $B_{2 g}$ symmetry that dominates, as in Ref. 13, with $A_{1 g}$ being subdominant. The order parameter of the dominant $B_{2 g}$ tendency is in Fig. 20 (b), while the subdominant $A_{1 g}$ is in Fig. 20.(c) (with the exotic detail that in this case the two hole pockets carry a different sign for the order parameter). The presence of the $B_{2 g}$ tendency was not only observed at the couplings of Fig. 20 and $n=4$, but also in a wide range of $J / U$ and varying the electronic density $n$ to 4.1 and 3.9 (not shown). Thus, such nodal pairing 
tendencies are robust. However, a more detailed analysis of the RPA response of the three-orbital model, motivated by the multiple wavevectors in its magnetic state, should be carried out in the future.

\section{CONCLUSIONS}

In this publication, the undoped limit of the multiorbital Hubbard model for pnictides has been studied using a standard mean-field approximation, similar to that employed in the study of the single-orbital Hubbard model for the undoped cuprates. Within this approximation, the magnitude of the order parameter associated with the $(\pi, 0)$ magnetic order was studied varying $U$ and $J / U$. In addition, the one-particle spectral function $A(\mathbf{k}, \omega)$ was also analyzed. Comparing results against neutron scattering and ARPES experiments, allow us to define regions in parameter space, dubbed "physical regions", where the mean-field model Hamiltonian predictions are in qualitative agreement with the above mentioned experiments. These regions are relatively small in size since the ground state in this regime must be simultaneously metallic, magnetic with order parameters in the range found by neutrons, and with Fermi surfaces containing satellite pockets induced by the magnetic state near the $\Gamma$-point hole pockets of the original band structure. Although fluctuations beyond the mean-field approximation are expected to enhance the physical regions, our results still provide important constraints on the couplings to be used for theoretical studies of multiorbital Hubbard models for pnictides.

In addition, in the regime of $U$ and $J / U$ described above, the RPA approximation allowed us to make predictions about the dominant pairing tendencies in the "physical regions". While it is clear that several channels are competing in these models, namely that small changes in parameters can lead to drastic changes in the dominant symmetries of the pairing states, the common property that emerges is the presence of nodal superconducting states in those physical regions. Within these models it appears very difficult to stabilize states without nodes. Even in regimes where the $A_{1 g}$ state dominates, it still has quasi-nodes (very small values of the amplitude at particular Fermi surface points) or true nodes. Thus, in these regards our results are more compatible with the bulk measurements that reported nodal superconductivity than with the ARPES experiments reporting nodeless superconductivity in doped pnictides. However, our studies are based on approximations that need to be refined. Hopefully, our study will initiate a debate on what are the true dominant tendencies in the multiorbital Hubbard model for pnictides, helping to decide if the mechanism is electronic or phononic.

\section{Acknowledgments}

This research was sponsored by the U.S. Department of Energy, Office of Basic Energy Sciences, Materials Sciences and Engineering Division (A.M. and E.D.), the Deutsche Forschungsgemeinschaft under the Emmy-Noether program
(M.D.), the Sun Yat-Sen University under the Hundred Talents program (D-X.Y.), and by the W. M. Keck Foundation (R.Y.). G.B.M. especially acknowledges the help of S. Graser in developing the RPA code, as well as discussions with A. Liebsch. E.D. acknowledges useful discussions with D. Scalapino.

\section{Appendix: parameters of five-orbital Model 1}

Using a similar notation as for three orbitals, and the parameters of Table II below, the tight-binding portion of the five-orbital Model 1 is $H_{\mathrm{TB}}(\mathbf{k})=\sum_{\mathbf{k}, \sigma, \mu, \nu} T_{\mu, \nu}(\mathbf{k}) d_{\mathbf{k}, \mu, \sigma}^{\dagger} d_{\mathbf{k}, v, \sigma}$, where

$$
\begin{aligned}
& T_{11 / 22}=2 t_{x / y}^{11} \cos k_{x}+2 t_{y / x}^{11} \cos k_{y}+4 t_{x y}^{11} \cos k_{x} \cos k_{y} \\
& \pm 2 t_{x x}^{11}\left(\cos 2 k_{x}-\cos 2 k_{y}\right)+4 t_{x x y / x y y}^{11} \cos 2 k_{x} \cos k_{y} \\
& +4 t_{x y y / x x y}^{11} \cos k_{x} \cos 2 k_{y}+4 t_{x x y y}^{11} \cos 2 k_{x} \cos 2 k_{y} \\
& +\epsilon_{11 / 22} \text {, } \\
& T_{33}=2 t_{x}^{33}\left(\cos k_{x}+\cos k_{y}\right)+4 t_{x y}^{33} \cos k_{x} \cos k_{y} \\
& +2 t_{x x}^{33}\left(\cos 2 k_{x}+\cos 2 k_{y}\right)+\epsilon_{33}, \\
& T_{44}=2 t_{x}^{44}\left(\cos k_{x}+\cos k_{y}\right)+4 t_{x y}^{44} \cos k_{x} \cos k_{y} \\
& +2 t_{x x}^{44}\left(\cos 2 k_{x}+\cos 2 k_{y}\right) \\
& +4 t_{x x y}^{44}\left(\cos 2 k_{x} \cos k_{y}+\cos k_{x} \cos 2 k_{y}\right) \\
& +4 t_{x x y y}^{44} \cos 2 k_{x} \cos 2 k_{y}+\epsilon_{44}, \\
& T_{55}=2 t_{x}^{55}\left(\cos k_{x}+\cos k_{y}\right)+2 t_{x x}^{55}\left(\cos 2 k_{x}+\cos 2 k_{y}\right) \\
& +4 t_{x x y}^{55}\left(\cos 2 k_{x} \cos k_{y}+\cos k_{x} \cos 2 k_{y}\right) \\
& +4 t_{x x y y}^{55} \cos 2 k_{x} \cos 2 k_{y}+\epsilon_{55}, \\
& T_{12}=T_{21} \\
& =-4 t_{x y}^{12} \sin k_{x} \sin k_{y} \\
& -4 t_{x x y}^{12}\left(\sin 2 k_{x} \sin k_{y}+\sin k_{x} \sin 2 k_{y}\right) \\
& -4 t_{x x y y}^{12} \sin 2 k_{x} \sin 2 k_{y} \text {, } \\
& T_{13 / 23}=\bar{T}_{31 / 32} \\
& = \pm 2 i t_{x}^{13} \sin k_{y / x} \pm 4 i t_{x y}^{13} \sin k_{y / x} \cos k_{x / y} \\
& \mp 4 i t_{x x y}^{13}\left(\sin 2 k_{y / x} \cos k_{x / y}-\cos 2 k_{x / y} \sin k_{y / x}\right) \text {, } \\
& T_{14 / 24}=\bar{T}_{41 / 42} \\
& =2 i t_{x}^{14} \sin k_{x / y}+4 i t_{x y}^{14} \sin k_{x / y} \cos k_{y / x} \\
& +4 i t_{x x y}^{14} \sin 2 k_{x / y} \cos k_{y / x}, \\
& T_{15 / 25}=\bar{T}_{51 / 52} \\
& =2 i t_{x}^{15} \sin k_{y / x}-4 i t_{x y}^{15} \sin k_{y / x} \cos k_{x / y} \\
& -4 i t_{x x y y}^{15} \sin 2 k_{y / x} \cos 2 k_{x / y} \text {, } \\
& T_{34}=T_{43} \\
& =4 t_{x x y}^{34}\left(\sin k_{x} \sin 2 k_{y}-\sin 2 k_{x} \sin k_{y}\right), \\
& T_{35}=T_{53} \\
& =2 t_{x}^{35}\left(\cos k_{x}-\cos k_{y}\right) \\
& +4 t_{x x y}^{35}\left(\cos 2 k_{x} \cos k_{y}-\cos k_{x} \cos 2 k_{y}\right) \text {, } \\
& T_{45}=T_{54} \\
& =4 t_{x y}^{45} \sin k_{x} \sin k_{y}+4 t_{x x y y}^{45} \sin 2 k_{x} \sin 2 k_{y} \text {. }
\end{aligned}
$$


TABLE II: Parameters for the tight-binding portion of the five-orbital Model 1 used here. The overall energy unit is electron volts.

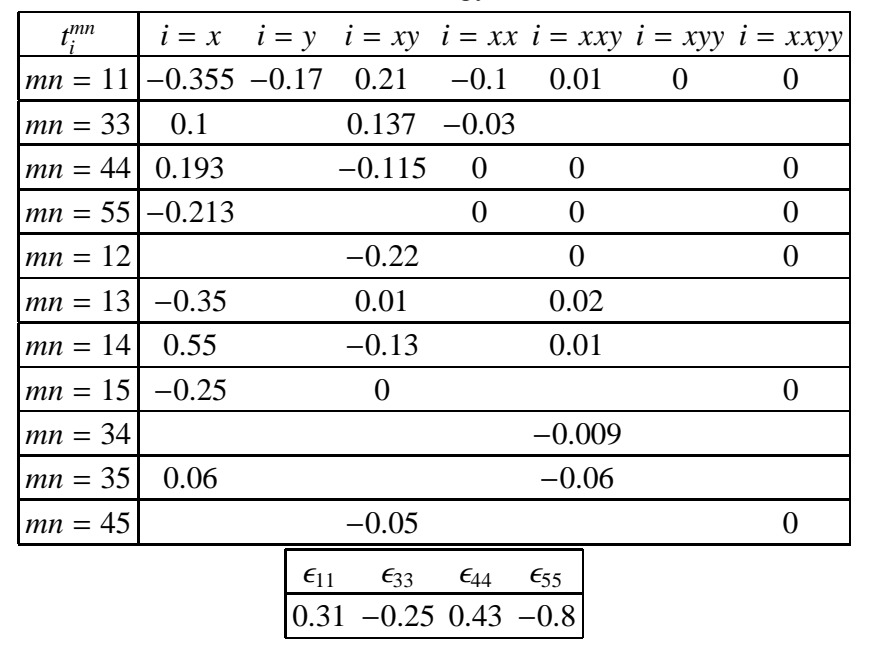

${ }^{1}$ Y. Kamihara, T. Watanabe, M. Hirano, and H. Hosono, J. of the Am. Chem. Soc. 130, 3296 (2008).

${ }^{2}$ G. F. Chen, Z. Li, G. Li, J. Zhou, D. Wu, J. Dong, W. Z. Hu, P. Zheng, Z. J. Chen, H. Q. Yuan, J. Singleton, J. L. Luo, and N. L. Wang, Phys. Rev. Lett. 101, 057007 (2008).

${ }^{3}$ G. F. Chen, Z. Li, D. Wu, G. Li, W. Z. Hu, J. Dong, P. Zheng, J. L. Luo, and N. L. Wang, Phys. Rev. Lett. 100, 247002 (2008).

${ }^{4}$ X. H. Chen, T. Wu, G. Wu, R. H. Liu, H. Chen, and D. F. Fang, Nature 453, 761 (2008).

5 Z.-A. Ren, W. Lu, J. Yang, W. Yi, X.-L. Shen, Z.-C. Li, G.-C. Che, X.-L. Dong, L.-L. Sun, F. Zhou, and Z.-X. Zhao, Chin. Phys. Lett. 25, 2215 (2008).

6 Z.-A. Ren, G.-C. Che, X.-L. Dong, J. Yang, W. Lu, W. Yi, X.L. Shen, Z.-C. Li, L.-L. Sun, F. Zhou, and Z.-X. Zhao, EPL 83, 17002 (2008).

7 D. C. Johnston, arXiv: 1005.4392

8 J. W. Lynn and P. Dai, Physica C 469, 469 (2009); and references therein.

9 M. D. Lumsden and A. D. Christianson, arXiv:1004.1969; and references therein.

10 C. Cao, P. J. Hirschfeld, and H.-P. Cheng, Phys. Rev. B 77, 220506(R) (2008); and references therein.

11 S. Raghu, X. L. Qi, C. X. Liu, D. J. Scalapino, and S. C. Zhang, Phys. Rev. B 77, 220503(R) (2008).

12 K. Kuroki, S. Onari, R. Arita, H. Usui, Y. Tanaka, H. Kontani, and H. Aoki, Phys. Rev. Lett. 101, 087004 (2008).

13 M. Daghofer, A. Moreo, J. A. Riera, E. Arrigoni, D. J. Scalapino, and E. Dagotto, Phys. Rev. Lett. 101, 237004 (2008).

14 M. M. Korshunov and I. Eremin, Phys. Rev. B 78, 140509(R) (2008).

15 Q. Si and E. Abrahams, Phys. Rev. Lett. 101, 076401 (2008).

16 K. Seo, B. A. Bernevig, and J. Hu, Phys. Rev. Lett. 101, 206404 (2008).

17 J. Lorenzana, G. Seibold, C. Ortix, and M. Grilli, Phys. Rev. Lett. 101, 186402 (2008).

18 R. Yu, K. T. Trinh, A. Moreo, M. Daghofer, J. A. Riera, S. Haas,
E. Dagotto, Phys. Rev. B 79, 104510 (2009).

19 R. Sknepnek, G. Samolyuk, Y.-bin Lee, and J. Schmalian, Phys. Rev. B 79, 054511 (2009).

20 A. Moreo, M. Daghofer, J. A. Riera, and E. Dagotto, Phys. Rev. B 79, 134502 (2009)

21 S. Graser, T. A. Maier, P. J. Hirschfeld, and D. J. Scalapino, New J. Phys. 11, 025016 (2009).

22 A. F. Kemper, T. A. Maier, S. Graser, H-P. Cheng, P. J. Hirschfeld, and D. J. Scalapino, New J. Phys. 12, 073030 (2010).

23 W.-Q. Chen, K.-Y. Yang, Y. Zhou, and F.-C. Zhang, Phys. Rev. Lett. 102, 047006 (2009).

${ }^{24}$ M. J. Calderón, B. Valenzuela, and E. Bascones, New J. Phys. 11, 013051 (2009).

25 E. Bascones, M. J. Calderón, and B. Valenzuela, Phys. Rev. Lett. 104, 227201 (2010).

${ }^{26}$ C.-C. Lee, W.-G. Yin, and W. Ku, Phys. Rev. Lett. 103, 267001 (2009).

27 P. A. Lee and X.-G. Wen, Phys. Rev. B 78, 144517 (2008).

${ }^{28}$ M. S. Laad and L. Craco, Phys. Rev. Lett. 103, 017002 (2009).

${ }^{29}$ Sen Zhou and Ziqiang Wang, arXiv:0910.2707

30 A. Moreo, M. Daghofer, A. Nicholson, and E. Dagotto, Phys. Rev. B 80, 104507 (2009).

31 M. Daghofer, A. Nicholson, A. Moreo, and E. Dagotto, Phys. Rev. B 81, 014511 (2010).

32 Xiaoyu Wang, Maria Daghofer, Andrew Nicholson, Adriana Moreo, Michael Guidry, and Elbio Dagotto, Phys. Rev. B 81, 144509 (2010).

${ }^{33}$ M. Daghofer, Q.-L. Luo, R. Yu, D. X. Yao, A. Moreo, and E. Dagotto, Phys. Rev. B 81, 180514(R)(2010).

34 J. R. Schrieffer, X. G. Wen, and S. C. Zhang, Phys. Rev. B 39, 11663 (1989).

35 A. M. Oleś, Phys. Rev. B 28, 327 (1983).

${ }^{36}$ L. X. Yang, Y. Zhang, H. W. Ou, J. F. Zhao, D. W. Shen, B. Zhou, J. Wei, F. Chen, M. Xu, C. He, Y. Chen, Z. D. Wang, X. F. Wang, T. Wu, G. Wu, X. H. Chen, M. Arita, K. Shimada, M. Taniguchi, Z. Y. Lu, T. Xiang, and D. L. Feng, Phys. Rev. Lett. 102, 107002 
(2009).

37 Y. Zhang, J. Wei, H. W. Ou, J. F. Zhao, B. Zhou, F. Chen, M. Xu, C. He, G. Wu, H. Chen, M. Arita, K. Shimada, H. Namatame, M. Taniguchi, X. H. Chen, and D. L. Feng, Phys. Rev. Lett. 102, 127003 (2009).

${ }^{38}$ M. Yi, D. H. Lu, J. G. Analytis, J.-H. Chu, S.-K. Mo, R.-H. He, M. Hashimoto, R. G. Moore, I. I. Mazin, D. J. Singh, Z. Hussain, I. R. Fisher, and Z.-X. Shen, Phys. Rev. B 80, 174510 (2009).

39 T. Shimojima, K. Ishizaka, Y. Ishida, N. Katayama, K. Ohgushi, T. Kiss, M. Okawa, T. Togashi, X. -Y. Wang, C. -T. Chen, S. Watanabe, R. Kadota, T. Oguchi, A. Chainani, and S. Shin, Phys. Rev. Lett. 104, 057002 (2010).

40 T. Kondo, R. M. Fernandes, R. Khasanov, C. Liu, A. D. Palczewski, Ni Ni, M. Shi, A. Bostwick, E. Rotenberg, J. Schmalian, S. L. Bud'ko, P. C. Canfield, and A. Kaminski, Phys. Rev. B 81, 060507(R) (2010).

${ }^{41}$ S. de Jong, E. van Heumen, S. Thirupathaiah, R. Huisman, F. Massee, J. B. Goedkoop, R. Ovsyannikov, J. Fink, H. A. Duerr, A. Gloskovskii, H.S. Jeevan, P. Gegenwart, A. Erb, L. Patthey, M. Shi, R. Follath, A. Varykhalov, and M. S. Golde, EPL 89, 27007 (2010).

42 D. Hsieh, Y. Xia, L. Wray, D. Qian, K. Gomes, A. Yazdani, G.F. Chen, J.L. Luo, N.L. Wang, and M.Z. Hasan, arXiv:0812.2289

${ }^{43}$ K. Hashimoto, T. Shibauchi, T. Kato, K. Ikada, R. Okazaki, H. Shishido, M. Ishikado, H. Kito, A. Iyo, H. Eisaki, S. Shamoto, and Y. Matsuda, Phys. Rev. Lett. 102, 017002 (2009).

44 T. Kondo, A. F. Santander-Syro, O. Copie, C. Liu, M. E. Tillman, E. D. Mun, J. Schmalian, S. L. Budko, M. A. Tanatar, P. C. Canfield, and A. Kaminski, Phys. Rev. Lett. 101, 147003 (2008).

45 H. Ding, P. Richard, K. Nakayama, K. Sugawara, T. Arakane, Y. Sekiba, A. Takayama, S. Souma, T. Sato, T. Takahashi, Z. Wang, X. Dai, Z. Fang, G. F. Chen, J. L. Luo, and N. L.Wang, EPL 83, 47001 (2008).

${ }^{46}$ C. Martin, R. T. Gordon, M. A. Tanatar, M. D. Vannette, M. E. Tillman, E. D. Mun, P. C. Canfield, V. G. Kogan, G. D. Samolyuk, J. Schmalian, and R. Prozorov, arXiv:0807.0876.

47 T. Y. Chen, Z. Tesanovic, R. H. Liu, X. H. Chen, and C. L. Chien, Nature (London) 453, 1224 (2008).

48 D. Parker, O. V. Dolgov, M. M. Korshunov, A. A. Golubov, and I. I. Mazin, Phys. Rev. B 78, 134524 (2008).

49 L. Shan, Y. Wang, X. Zhu, G. Mu, L. Fang, C. Ren, and H.-H. Wen, EPL 83, 57004 (2008).

${ }^{50}$ M. Gang, Z. Xi-Yu, F. Lei, S. Lei, R. Cong, and W. Hai-Hu, Chin. Phys. Lett. 25, 2221 (2008).

51 C. Ren, Z.-S. Wang, H. Yang, X. Zhu, L. Fang, G. Mu, L. Shan, and H.-H. Wen, arXiv:0804.1726

${ }^{52}$ K. Ahilan, F. L. Ning, T. Imai, A. S. Sefat, R. Jin, M. A. McGuire, B. C. Sales, and D. Mandrus, Phys. Rev. B 78, 100501(R) (2008).

53 Y. Nakai, K. Ishida, Y. Kamihara, M. Hirano, and H. Hosono, J. Phys. Soc. Jpn. 77, 073701 (2008).

${ }^{54}$ H.-J. Grafe, D. Paar, G. Lang, N. J. Curro, G. Behr, J. Werner, J. Hamann-Borrero, C. Hess, N. Leps, R. Klingeler, and B. Büchner, Phys. Rev. Lett. 101, 047003 (2008).
55 K. Matano, Z. A. Ren, X. L. Dong, L. L. Sun, Z. X. Zhao, and Guo-qing Zhen, EPL 83, 57001 (2008).

${ }^{56}$ H. Mukuda, N. Terasaki, H. Kinouchi, M. Yashima, Y. Kitaoka, S. Suzuki, S. Miyasaka, S. Tajima, K. Miyazawa, P. Shirage, H. Kito, H. Eisaki, and A. Iyo, J. Phys. Soc. Jpn. 77, 093704 (2008).

57 O. Millo, I. Asulin, O. Yuli, I. Felner, Z.-A. Ren, X.-L. Shen, G.C. Che, and Z.-X. Zhao, Phys. Rev. B 78, 092505 (2008).

58 X. L. Wang, S. X. Dou, Z.-A. Ren, W. Yi, Z.-C. Li, Z.-X. Zhao, and S.-I. Lee, arXiv:0808.3398

59 K. Hashimoto, A. Serafin, S. Tonegawa, R. Katsumata, R. Okazaki, T. Saito, H. Fukazawa, Y. Kohori, K. Kihou, C. H. Lee, A. Iyo, H. Eisaki, H. Ikeda, Y. Matsuda, A. Carrington, and T. Shibauchi, arXiv:1003.6022, and references therein.

${ }^{60}$ B. Zeng, G. Mu, B. Shen, P. Cheng, H. Luo, H. Yang, L. Shan, C. Ren, and H-H. Wen, arXiv:1006.2785

${ }^{61}$ For a discussion of this relation in the manganite context see E. Dagotto, T. Hotta, and A. Moreo, Phys. Rep. 344, 1 (2001) and references therein.

62 T. Nomura and K. Yamada, J. Phys. Soc. Jpn. 69, 1856 (2000).

${ }^{63}$ Other estimations of $U$ and $J$ have been presented in the literature. In T. Miyake, K. Nakamura, R. Arita, and M. Imada, J. Phys. Soc. Jpn. 79, 044705 (2010), a combination of the constrained randomphase approximation and the maximally localized Wannier function gives $J / U \sim 0.14$ and $U \sim 2.8 \mathrm{eV}$. Other investigations, such as those based on dynamical mean-field theory presented by $\mathrm{H}$. Ishida and A. Liebsch, Phys. Rev. B 81, 054513 (2010) also report a $U \sim 3 \mathrm{eV}$, and $J / U \sim 0.25$. While these values of $J / U$ are at or close to the lower range of our "physical regions", the value of $U$ differs from ours by approximately a factor 3 for the three-orbital model and 2 for the five-orbital models. However, note that the approaches cited above and ours are fundamentally different. In the first case, estimations for $J$ and $U$ are obtained directly based on ab-initio calculations, while in our approach it is the comparison with experiments and the solution of the model via mean-field techniques that are employed. The reason for this discrepancy in $U$ remains to be investigated.

${ }^{64}$ Note that the results of Ref. 21 were obtained at a chemical potential $\mu=0$, which corresponds to a slightly different electronic density $n=6.05$.

65 K. Kuroki, H. Usui, S. Onari, R. Arita, and H. Aoki, Phys. Rev. B 79, 224511 (2009).

${ }^{66}$ R. Berk and J. R. Schrieffer, Phys. Rev. Lett. 14, L369 (1966).

${ }^{67}$ D. J. Scalapino, E. Loh, and J. Hirsch, Phys. Rev. B 34, 8190 (1986).

68 L. Puig-Puig, F. Lopez-Aguilar, and J. Costa-Quintana, Journ. Phys.-Cond. Matt. 6, 4929 (1994).

${ }^{69}$ B. Muschler, W. Prestel, R. Hackl, T. P. Devereaux, J. G. Analytis, Jiun-Haw Chu, and I. R. Fisher, Phys. Rev. B 80, 180510 (2009).

${ }^{70}$ N. Bulut, D. J. Scalapino, and S. R. White, Phys. Rev. B 47, 14599 (1993).

71 M. Vojta and E. Dagotto, Phys. Rev. B 59, R713 (1999). 